\title{
Topology optimization of 2D in-plane single mass MEMS gyroscopes
}

\author{
Giannini, Daniele; Braghin, Francesco ; Aage, Niels
}

Published in:

Structural and Multidisciplinary Optimization

Link to article, DOI:

10.1007/s00158-020-02595-3

Publication date:

2020

Document Version

Peer reviewed version

Link back to DTU Orbit

Citation (APA):

Giannini, D., Braghin, F., \& Aage, N. (2020). Topology optimization of 2D in-plane single mass MEMS gyroscopes. Structural and Multidisciplinary Optimization, 62, 2069-2089. https://doi.org/10.1007/s00158-02002595-3

\section{General rights}

Copyright and moral rights for the publications made accessible in the public portal are retained by the authors and/or other copyright owners and it is a condition of accessing publications that users recognise and abide by the legal requirements associated with these rights.

- Users may download and print one copy of any publication from the public portal for the purpose of private study or research.

- You may not further distribute the material or use it for any profit-making activity or commercial gain

- You may freely distribute the URL identifying the publication in the public portal

If you believe that this document breaches copyright please contact us providing details, and we will remove access to the work immediately and investigate your claim 


\title{
Topology optimization of 2D in-plane single mass MEMS gyroscopes
}

\author{
Daniele Giannini · Francesco Braghin • Niels Aage
}

Received: date / Accepted: date

\begin{abstract}
In this paper we apply the topology optimization method to the design of MEMS gyroscopes, with the aim of supporting traditional trial and error design procedures. Using deterministic, gradient based mathematical programming, the approach is here applied to the design of 2D in-plane single mass MEMS gyroscopes.

We first focus on a benchmark academic case, for which we present and compare three different formulations of the optimization problem, considering typical industrial requirements. These include the maximization of the response of the sensor's structure to the external angular rate, target resonant frequencies and minimum or constrained material usage. Also, a minimum length scale is imposed to the geometric features in order to ensure manufacturability, and an explicit penalization of grey elements is proposed to improve convergence to black and white layouts.

Once the suitability of the method has been assessed, the formulation associated with the lowest computational cost, i.e. the one considering static estimations of the resonant frequencies, is applied to the design of a real-world MEMS gyroscope, targeting different resonant frequencies.
\end{abstract}

Keywords Topology optimization · Single mass MEMS gyroscopes · eigenfrequencies tuning · harmonic response $\cdot$ Coriolis force $\cdot$ minimum length scale

D. Giannini (https://orcid.org/0000-0002-1214-2570) F.Braghin (https://orcid.org/0000-0002-0476-4118) Department of Mechanical Engineering, Politecnico di Milano, Via G. La Masa 1, 20156 Milano (MI), Italy

N. Aage (https://orcid.org/0000-0002-3042-0036 Centre for Acoustic-Mechanical Micro Systems, Department of Mechanical Engineering, Technical University of Denmark, Nils Koppels Alle, Building 404, DK-2800 Kgs. Lyngby, Denmark

Corresponding author: daniele.giannini@polimi.it

(Daniele Giannini)

\section{Introduction}

The market of inertial Micro Electro Mechanical Systems (MEMS) has grown at a spectacular rate in the last two decades. This kind of devices includes sensors such as accelerometers and gyroscopes, originally developed for military and space-related utilities but now found in a multitude of everyday products. For example, airbag-release sensors and electronic stability control are now standard in all new cars. Also, activity monitoring of pacemaker patients and stabilization of platforms such as transport robots, drones and cameras are now improving our quality of life through the use of inertial MEMS devices. But, probably, the most interesting application is the motion sensing integrated into consumer electronics (mobile phones, game controllers, toys, etc.), personal navigation systems and other human-machine interfaces. The introduction of inertial MEMS into new application areas is a trend that is still gaining momentum Kempe, 2011. Potential markets of growth are expected through the evolution of sensors for wearable electronics, mobile healthcare, and Internet of Things (IoT).

In particular, MEMS gyroscopes are inertial sensors that are used to detect external angular velocities. Even though an extensive variety of MEMS gyroscope designs and operation principles exist, the majority of the reported micromachined gyroscopes use vibrating mechanical elements to sense angular rate Acar and Shkel, 2009]. The gyroscope is usually a microresonator with two resonant modes, the drive mode and the sense mode. The resonator will vibrate at its drive resonant mode with a constant frequency and amplitude when excited by electrostatic, electromagnetic, piezoelectric or other forces. The angular rate in the secondary mode direction can be detected because of 
the Coriolis force coupling the two modes Xia et al., 2014 .

In recent years the increasing performance requirements have led to a higher and higher complexity in the mechanical structure of MEMS gyroscopes Xia et al., 2014. From the classical tuning fork structures able to reject common mode inputs Bernstein et al., 1993. Zaman et al., 2006, many categories of gyroscopes have appeared. Structurally decoupled gyroscopes Acar and Shkel, 2005, Kulygin et al., 2012 minimize the undesired mechanical coupling between the drive mode and the sense mode. Multi degree of freedom gyroscopes Acar and Shkel, 2001, Sahin et al., 2009 increase the robustness against variations in the structural parameters. Dual-axis and multi-axis gyroscopes Trusov et al., 2011, Sung et al., 2011 allow to lower the costs while still increasing the efficiency of Inertial Measurement Units (IMUs).

The mechanics of MEMS devices are typically designed using a set of popular building blocks (rectangular cantilevers, folded flexure beams, crab legs, etc.), combined with scaling laws and/or engineering insight gained from studying the relationships between geometric features and the device behaviour. In general, the design of MEMS devices relies on a trial and error approach ("Build and Break") dependent on the designer experience. This is both time-consuming and expensive Benkhelifa et al., 2010a, Farnsworth et al., 2017.

As the complexity in the mechanical structure increases, the design procedure of the devices becomes more and more crucial. For example, Xia et al., 2015 shows the issues related to the design of a beatingheart triaxial gyroscope. Such type of sensor, originally introduced by the commercial company STMicroelectronics Vigna, 2011, exploits a complex triple tuning fork structure with a single drive mode and three sense modes, one for each detection axis. In this case the designer has to tune the geometries and the features of all the mechanical elements of the gyroscope in order to match the desired target resonant frequencies. The result is a long and laborious procedure.

Therefore, the development of a design procedure that allows to improve the process of modeling, simulation and optimization of inertial MEMS devices is fundamental to ensure progress in the industry Benkhelifa et al., 2010a. Work on MEMS design automation and optimization consists mainly of sizing existing layouts. That is, the layout of the structure is decomposed into basic mechanical elements, such as masses, beams, plates and compliant parts. As in Xia et al., 2015 or Benkhelifa et al., 2010b, the shape and connectivity between the elements is fixed, while their size and location are used as design variables. These parameters are then optimized by means of numerical methods for parametric optimization: examples include both gradient-based methods Haronian, 1995] and stochastic or evolutionary algorithms [Xia et al., 2015, Benkhelifa et al., 2010b, Chen et al., 2012, Solouk et al., 2019].

Another interesting approach for structural optimization is topology optimization. This method does not require any initial definition and parametrization of the layout by the designer, but directly looks for the best way to distribute the material in the available design space. This approach therefore systematically generates the full topology of the design, including the shape, size, and location of features. The technique was first introduced for the minimization of mechanical compliance of structural components Bendsoe and Sigmund, 2004 subjected to a constraint on the available material. The method has since then matured such that it is no longer confined to single components Aage et al., 2017, and is now regularly being used by private companies such as Airbus and Boeing Krog et al., 2004. Beyond the traditional minimum compliance problem, the approach has been employed to solve a broad range of multiphysical problems Bendsoe and Sigmund, 2004, including some in the MEMS domain, e.g. electro-thermal-mechanical actuators and various piezo based devices [Sigmund, 2001, Sardan et al., 2008, Philippine et al., 2013, Bruggi et al., 2016]. The focus of the present work is on dynamics and vibrations, for which the first papers on frequency optimization can be found in Olhoff, 1989, Díaaz and Kikuchi, 1992, Tenek and Hagiwara, 1993, Ma et al., 1995 and the seminal work on dynamic compliance optimization can be found in [Jog, 2002, Tcherniak, 2002. Specific issues related to dynamic problems are managing multiple eigenvalues as treated in Seyranian et al., 1994], and selecting appropriate interpolation schemes, for which a thorough discussion is presented in Stolpe and Svanberg, 2001, Pedersen, 2000. Finally, this work will utilize target frequency constraints building on the findings of Ma et al., 1994.

The aim of this paper is to apply topology optimization to the design of MEMS gyroscopes. In particular, a $2 \mathrm{D}$ structure representing the layout of a single mass monoaxial gyroscope is considered. The technique is applied to optimize the elastic parts of the structure in order to match the prescribed target eigenfrequencies and to maximize the response of the sensor to the external angular rate. In order to ensure the manufacturability of the optimized layout, a minimum thickness constraint for the geometric features is imposed.

The paper is organized as follows: in Section 2 the general objectives and requirements regarding the de- 
sign of MEMS gyroscopes are introduced. Section 3 presents the physical models used to study the dynamics of the MEMS gyroscope and introduces the design variables of the topology optimization process. Three possible formulations of the optimization problem are described in Section 4, while Section 5 presents the proposed topology optimization algorithm. In Section 6 . the different formulations are applied and compared for a benchmark academic design case, and in Section 7 the simplest of the optimization formulations, i.e. the one exploiting a static estimation of the resonant frequencies, is applied to a real MEMS gyroscope design case, considering different target resonant frequencies. Finally, Section 8 concludes the paper with a summary of the obtained results and a view of the interesting points to be investigated in the future research.

\section{Problem statement}

A simple, yet representative, model of a MEMS vibratory gyroscope is illustrated in Fig. 1. The system consists of a proof mass suspended by a compliant structure mounted on an external box that acts as a support frame. The suspending structure allows the mass to displace in two orthogonal directions: the drive direction along the $\mathrm{x}$-axis and the sense direction along the y-axis. A harmonic oscillation is imposed along the drive direction, usually through forcing electrodes and a control loop. When the gyroscope is subjected to an external angular rate $\Omega_{z}$, a sinusoidal Coriolis force, proportional in amplitude to $\Omega_{z}$, is induced at the frequency of the drive oscillation along the sense direction. The Coriolis force excites the sense mode, causing the proof-mass to displace along the sense direction. Such sinusoidal displacement, proportional to the generated Coriolis force and thus to the external angular rate $\Omega_{z}$, is picked up by the detection electrodes.

The purpose of the suspending structure design is to provide two well-controlled modes of oscillations for the drive and the sense directions, and to prevent other movements of the mass, such as in-plane rotations. Fig. 2 schematizes the main requirements for the resonant frequencies of the gyroscope. In particular, the drive mode resonant frequency $\omega_{d}$ is usually chosen to be higher than the bandwidth of acoustic noise $(>10$ $\mathrm{kHz}$ ). In order to achieve the maximum possible gain in the Coriolis response, it is generally desirable to operate the gyroscope at, or near, the peak of the sense-mode. This is typically achieved by matching the sense resonant frequency $\omega_{s}$ with the drive one $\omega_{d}$, that greatly enhances the sense mode mechanical response to the angular rate input. However, operating close to the sense resonant peak makes the system very sensitive

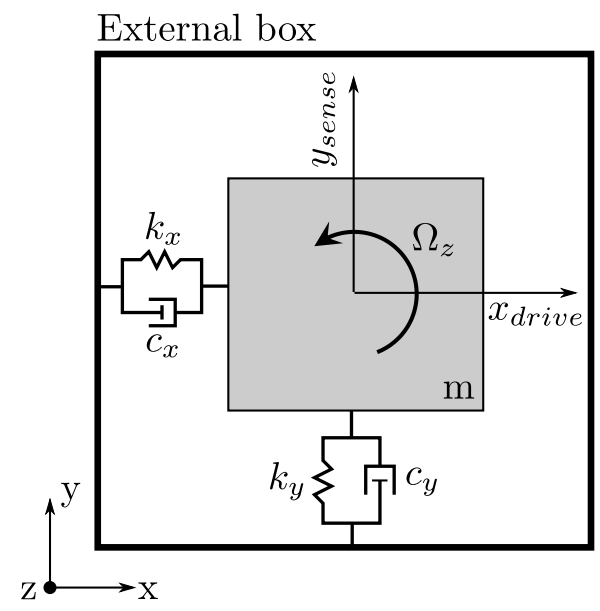

Fig. 1: Simple model of a MEMS vibratory gyroscope.

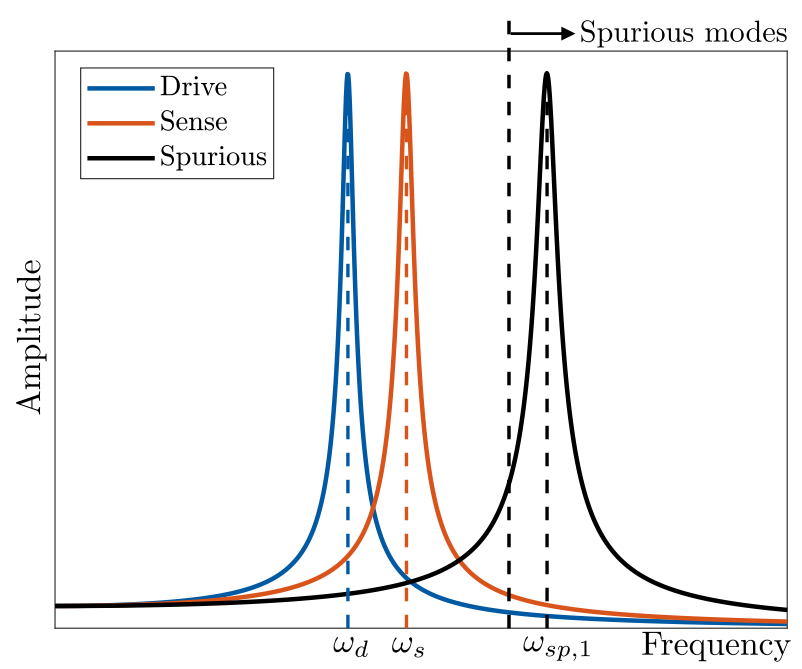

Fig. 2: Main requirements for the resonant frequencies of MEMS gyroscopes.

to variations in system parameters which can cause a shift in the resonant frequencies or damping properties. Thus, the sense resonant frequency $\omega_{s}$ is set around $2-10 \%$ higher than the drive-mode frequency $\omega_{d}$ Acar and Shkel, 2009. In addition all other so-called "spurious" resonant frequencies $\omega_{s p, i}$, associated for example to in-plane rotations of the mass or to the internal dynamics of the suspending structure, have to be kept far enough from the sense one. In the following, we will identify angular frequencies with the letter $\omega[\mathrm{rad} / \mathrm{s}]$ and the corresponding frequencies with $f=\omega / 2 \pi[\mathrm{Hz}]$.

Fig. 3 illustrates the focus of the present work, in which the objective is to design the suspending structure in a certain design space around a proof mass $m$, 


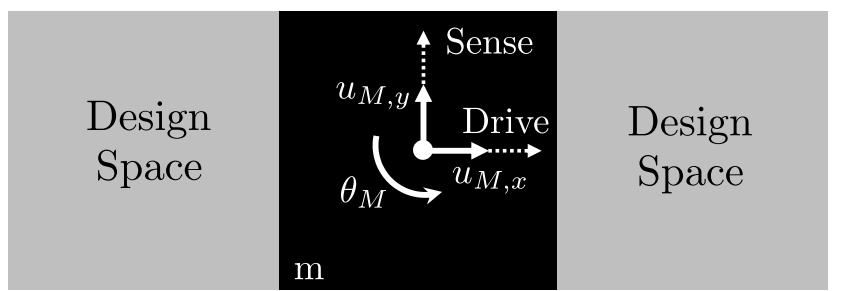

Fig. 3: Scheme of the presented design problem.

to maximize the sense response and to match the requirements on the target resonant frequencies.

\section{Physical model}

\subsection{Dynamics of the MEMS gyroscope}

In this paper the MEMS gyroscope is modeled as a $2 \mathrm{D}$ planar structure: for simplicity the focus is here on the in-plane linear dynamics of the gyroscope, while the out-of-plane motion is not treated. The main assumptions for the elastic constitutive laws are therefore small strains and a linear and isotropic plane stress condition.

Considering time-harmonic motion and a discretization of the domain by linear Q4 Lagrange finite elements Cook, 1995, the eigenfrequencies of the structure $\omega_{i}$ and the corresponding mode shapes $\boldsymbol{\Phi}_{\boldsymbol{i}}$ can be computed by solving the following undamped eigenvalue problem:

$$
\left(-\omega_{i}^{2} \boldsymbol{M}+\boldsymbol{K}\right) \boldsymbol{\Phi}_{\boldsymbol{i}}=\mathbf{0}
$$

In Eq. (1), $\boldsymbol{M}$ and $\boldsymbol{K}$ are the global mass and stiffness matrices of the structure, obtained through the assembly of the elements matrices $\boldsymbol{M}_{\boldsymbol{e}}$ and $\boldsymbol{K}_{\boldsymbol{e}}$, given by:

$$
\begin{gathered}
\boldsymbol{K}=\sum_{e} \boldsymbol{K}_{\boldsymbol{e}}=\sum_{e} \int_{\Omega_{e}} E_{e} \boldsymbol{B}_{\boldsymbol{u}}{ }^{T} \boldsymbol{C} \boldsymbol{B}_{\boldsymbol{u}} d \Omega_{e} \\
\boldsymbol{M}=\sum_{e} \boldsymbol{M}_{\boldsymbol{e}}=\sum_{e} \int_{\Omega_{e}} \rho_{e} \boldsymbol{N}_{\boldsymbol{u}}{ }^{T} \boldsymbol{N}_{\boldsymbol{u}} d \Omega_{e}
\end{gathered}
$$

Here $E_{e}$ and $\rho_{e}$ are the Young modulus and the mass density of the material inside the $e$-th element. $\boldsymbol{N}_{\boldsymbol{u}}$ is the shape functions matrix for the displacements defined inside each element domain $\Omega_{e}, \boldsymbol{B}_{\boldsymbol{u}}$ is the linear strain-displacement matrix computed through the differential operator $\boldsymbol{\partial}$ and $\sum_{e}$ is intended as the standard finite element assembly operator. The matrix $\boldsymbol{C}$ is used to express the plane stress material constitutive relationship:

$\boldsymbol{B}_{\boldsymbol{u}}=\boldsymbol{\partial} \boldsymbol{N}_{\boldsymbol{u}}$
$\boldsymbol{\partial}=\left[\begin{array}{cc}\frac{\partial}{\partial x} & 0 \\ 0 & \frac{\partial}{\partial y} \\ \frac{\partial}{\partial y} & \frac{\partial}{\partial x}\end{array}\right]$

$\boldsymbol{C}=\frac{1}{1-\nu^{2}}\left[\begin{array}{ccc}1 & \nu & 0 \\ \nu & 1 & 0 \\ 0 & 0 & \frac{1-\nu}{2}\end{array}\right]$

where $\nu$ is the Poisson ratio of the material.

The response of the gyroscope to the external angular rate $\Omega_{z}$ can be computed by imposing an harmonic drive displacement in correspondence of the horizontal degree of freedom of the center of gravity of the proof mass $u_{M, x}$ (cf. Fig. 3), and solving for the steady-state response of the structure. The nodal vector of harmonic displacements $\boldsymbol{u}_{\boldsymbol{h}}$ can be obtained by solving the following system of equations:

$\left(-\omega_{h}^{2} \boldsymbol{M}+i \omega_{h} \boldsymbol{G}_{\boldsymbol{C}}\left(\Omega_{z}\right)+\boldsymbol{K}\right) \boldsymbol{u}_{\boldsymbol{h}}=\boldsymbol{f}_{\boldsymbol{h}}$

In Eq. (4), $\omega_{h}$ is the frequency of excitation and the vector of harmonic loads $\boldsymbol{f}_{\boldsymbol{h}}$ contains the reaction forces associated to the imposed drive displacement and to the constrained degrees of freedom. In the following, special focus will be given to the response of the proof mass along the sense direction, namely to the vertical degree of freedom $u_{M, y}$ (cf. Fig. 3).

Damping in MEMS gyroscopes is usually kept very low (adimensional damping ratios are in the order of $\left.10^{-3}\right)$ : it is therefore possible to neglect its effect on resonant frequencies shifts, and also the dynamic response when a proper mismatch between the drive and sense resonant frequencies is imposed Acar and Shkel, 2009. The effect of the Coriolis forces due to the external angular rate $\Omega_{z}$ is instead included in the skewsymmetric matrix $\boldsymbol{G}_{\boldsymbol{C}}\left(\Omega_{z}\right)$, that is assembled from the element matrices $\boldsymbol{G}_{\boldsymbol{C}, \boldsymbol{e}}\left(\Omega_{z}\right)$ :

$$
\begin{aligned}
\boldsymbol{G}_{\boldsymbol{C}}\left(\Omega_{z}\right) & =\sum_{e} \boldsymbol{G}_{\boldsymbol{C}, \boldsymbol{e}}\left(\Omega_{z}\right)= \\
& =\sum_{e} \int_{\Omega_{e}} 2 \rho_{e} \boldsymbol{N}_{\boldsymbol{u}}{ }^{T} \boldsymbol{\Omega}\left(\Omega_{z}\right) \boldsymbol{N}_{\boldsymbol{u}} d \Omega_{e}
\end{aligned}
$$

where $\sum_{e}$ is again intended as the standard finite element assembly operator, and $\boldsymbol{\Omega}\left(\Omega_{z}\right)$ is a skew-symmetric matrix function of the external angular rate $\Omega_{z}$ :

$\boldsymbol{\Omega}\left(\Omega_{z}\right)=\left[\begin{array}{cc}0 & -\Omega_{z} \\ \Omega_{z} & 0\end{array}\right]$ 
We note also that the focus of the analysis is only on the steady-state dynamics of the gyroscope, while the transient homogeneous response has been neglected. This follows typical practice in the study of these sensors Acar and Shkel, 2009]: a steady-state drive motion is guaranteed by control systems, while the sense motion is assumed to be forced by an external angular rate at sufficiently low frequency with respect to the structure sense dynamics. Also, other harmonics than the one at the drive frequency are usually filtered by proper demodulation of the sense signal.

The FEM solver is implemented in MATLAB: the eigenvalue problem in Eq. (1) is solved by ARPACK (as "eigs"), applying an Implicitly Restarted Lanczos Method (IRLM), whereas the harmonic response in Eq. (4) is obtained by UMFPACK (as "\"), applying LU decomposition.

\subsection{Design variables of the topology optimization problem}

The topology optimization problem is formulated using the density method, which is a pixel based description of the structural layout. One design variable is assigned to each finite element and denoted by $\gamma_{e}$. This set of variables allows to describe the material distribution in the design space, scaling the properties of each finite element between solid and void.

In order to obtain a smooth optimization problem, the design variables are allowed to vary from 0 to 1 , where 0 corresponds to void and 1 to solid. Intermediate values are permitted during the optimization process, but are penalized in order to ensure all elements are 0 or 1 in the final design. This is achieved by properly designing the interpolation functions of the material properties, and combining them with an appropriate regularization of the optimization problem through filtering/projection techniques and length-scale control (cf. Section 3.3) Bourdin, 2001, Bruns and Tortorelli, 2001, Guest et al., 2004, Wang et al., 2011.

\subsection{Regularization}

The filtering and projection scheme from Wang et al., 2011 is adopted throughout the presented work and described in the following Section. Starting from the design variables $\gamma_{e}$, the filtered variables $\tilde{\gamma}_{e}$ are obtained by a weighted average of the neighbouring elements $\gamma_{e}$, i.e. through a convolution type filter:

$\tilde{\gamma}_{e}=\frac{\sum_{j \in \mathbb{N}_{s, e}} w\left(x_{j}\right) v_{j} \gamma_{e, j}}{\sum_{j \in \mathbb{N}_{s, e}} w\left(x_{j}\right) v_{j}}$

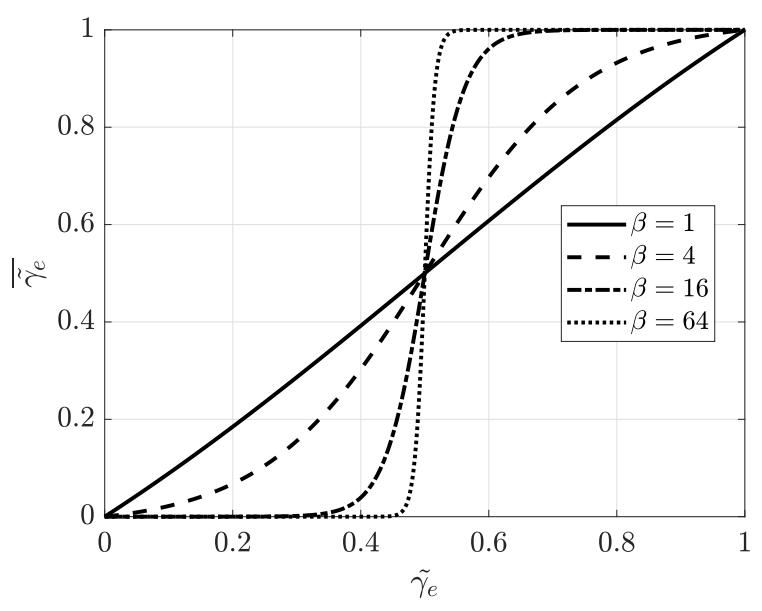

Fig. 4: Projection filter scheme (Eq. (9)), for threshold $\eta=0.5$ and different values of the sharpness parameter $\beta$.

where $v_{j}$ is the volume of the $j$-th element and $\mathbb{N}_{s, e}$ is the set of neighbouring elements, defined as the set of elements lying within a circle with radius $r_{\text {min }}$ centered on element $e$. The linear weighting function $w\left(x_{j}\right)$ is given as:

$w\left(x_{j}\right)=r_{\min }-\left|x_{j}-x_{e}\right|$

A threshold projection filter ensures convergence towards a solid/void solution. The projected design variables (or physical densities) $\overline{\tilde{\gamma}}_{e}$ are Whang et al., 2011:

$\overline{\tilde{\gamma}}_{e}=\frac{\tanh (\beta \eta)+\tanh \left(\beta\left(\tilde{\gamma}_{e}-\eta\right)\right)}{\tanh (\beta \eta)+\tanh (\beta(1-\eta))}$

where $\beta$ is the sharpness parameter controlling the slope of the smoothed Heaviside function and $\eta$ is the location of the threshold. The threshold is set to $\eta=$ 0.5 , while $\beta$ is increased incrementally such that the design gradually converges to a solid/void solution (cf. Section 6.2. Fig. 4 plots the projection scheme for several values of $\beta$.

The projected design variables are then used to interpolate the material properties of the elements between solid and void.

\subsection{Interpolation of material properties}

In this work a Rational Approximation of Material Properties (RAMP) model is used to interpolate the stiffness properties Stolpe and Svanberg, 2001], while a linear interpolation is used for the mass properties. 
The Young modulus $E_{e}$ and the mass density $\rho_{e}$ for a generic element $e$ with physical density $\overline{\tilde{\gamma}}_{e}$ will be:

$E_{e}=E_{m i n}+\frac{\overline{\tilde{\gamma}}_{e}}{1+q\left(1-\overline{\tilde{\gamma}}_{e}\right)}\left(E_{0}-E_{\text {min }}\right)$

$\rho_{e}=\rho_{\min }+\overline{\tilde{\gamma}}_{e}\left(\rho_{0}-\rho_{\text {min }}\right)$

where $E_{0}$ and $\rho_{0}$ are the Young modulus and mass density of the considered material, while $E_{\min }=10^{-8}$. $E_{0}$ and $\rho_{\min }=10^{-7} \cdot \rho_{0}$ are small values assigned to void elements, in order to avoid singular element matrices $\boldsymbol{K}_{\boldsymbol{e}}, \boldsymbol{M}_{\boldsymbol{e}}$ and $\boldsymbol{G}_{\boldsymbol{C}, \boldsymbol{e}}\left(\Omega_{z}\right)$ (Eq. (2) and (5)). In the following, we will first consider normalized Young modulus and mass density for a benchmark design case (cf. Section 60, and then we will use the actual material properties of polysilicon (cf. Section 7).

The choice of a nonlinear interpolation for stiffness is made to ensure convergence towards black and white final designs. This is also a reasonable choice because the intermediate densities can in fact be realized, or at least interpreted, following the Hashin-Shtrikman bounds Bendsøe and Sigmund, 1999. The linear interpolation of the mass densities is used because this is the only choice that makes physical sense following the rule of mixtures. We also note that combining the classical SIMP with a linear density interpolation often leads to numerical artifacts, which further motivates the use of the RAMP type interpolation for the stiffness Pedersen, 2000. In particular, we use $q=4$ in Eq. 10 for the RAMP interpolation.

\section{Formulation of the optimization problems}

Since a systematic study of topology optimization for MEMS gyroscopes has not been presented before, we propose, investigate and compare three different possible formulations. Besides including both the response to the external angular rate and the structure eigenfrequencies in the optimization problem, we in fact investigate the degree to which the problem can be simplified and still provide good and well-performing designs, proposing two levels of simplification.

The first design problem is to optimize the structure in order to maximize its sense response to the external angular rate and to match the target values of the resonant frequencies. This requires the solution of two finite element problems, i.e. an eigenfrequency problem as well as a subsequent forced response which depends on the eigenfrequency solution. In the second problem, the structure is optimized to minimize the material usage and we only require the design to match the target resonant frequencies, i.e. only an eigenfrequency problem needs to be solved. The third and final problem represents a simplification of the second design problem, employing a static estimation of the eigenfrequencies in order to minimize the associated computational cost.

4.1 (P1) Maximum sense response with target eigenfrequencies

The first topology optimization problem (P1), based on all the required physics, can be stated as seen in Eq. (11). The motivation for the choice of objective and constraints will be explained in details in the following Section.

$$
\begin{array}{ll}
\max _{\gamma_{e}} & \Psi_{1}=\left(\frac{\psi_{1}}{\psi_{1}^{(i t=0)}}\right)^{2} \\
\text { subject to } & \frac{\left(\omega_{d}-\omega_{d, d e s}\right)^{2}}{\omega_{d, d e s}^{2}}-\varepsilon^{2} \leq 0 \\
& \frac{\left(\omega_{s}-\omega_{s, d e s}\right)^{2}}{\omega_{s, d e s}^{2}}-\varepsilon^{2} \leq 0 \\
& \frac{1.4 \cdot \omega_{s}}{\omega_{s p}}-1 \leq 0 \\
& \omega_{d, d e s}=0.95 \cdot \omega_{s, d e s} \\
& \left(-\omega_{i}^{2} \boldsymbol{M}+\boldsymbol{K}\right) \boldsymbol{\Phi}_{\boldsymbol{i}}=\mathbf{0}, i=1 \ldots n_{\omega} \\
& \left(-\omega_{h}^{2} \boldsymbol{M}+i \omega_{h} \boldsymbol{G}_{\boldsymbol{C}}\left(\Omega_{z}\right)+\boldsymbol{K}\right) \boldsymbol{u}_{\boldsymbol{h}}=\boldsymbol{f}_{\boldsymbol{h}} \\
& \omega_{h}=\alpha \cdot \omega_{s}=0.95 \cdot \omega_{s} \\
& \text { Vol }=\frac{\sum_{e} \overline{\tilde{\gamma}}_{e} v_{e}}{\sum_{e} v_{e}} \leq v^{*} \\
& \mathrm{Geometric} \mathrm{constraints}
\end{array}
$$

Grey penalization constraints

In (P1) the objective function $\Psi_{1}$ is set to maximize the square of the sense response $\psi_{1}$, normalized with respect to its value $\psi_{1}^{(i t=0)}$ at the initial iteration. The sense response $\psi_{1}$ is represented by the modulus of the transfer function between the imposed drive displacement $u_{x, M}$ and the sense displacement $u_{y, M}$ due the external angular rate (cf. Fig. 3). Namely:

$\psi_{1}=\left\|\frac{u_{y, M}}{u_{x, M}}\right\|=\left\|\frac{\boldsymbol{l}_{\boldsymbol{y}}^{T} \boldsymbol{u}_{\boldsymbol{h}}}{\boldsymbol{l}_{\boldsymbol{x}}^{T} \boldsymbol{u}_{\boldsymbol{h}}}\right\|$

These components can be extracted from the solution of the harmonic response analysis (Eq. (4) $) \boldsymbol{u}_{\boldsymbol{h}}$ through the vectors $\boldsymbol{l}_{\boldsymbol{y}}$ and $\boldsymbol{l}_{\boldsymbol{x}}$. 
Constraints are instead imposed to match the target eigenfrequencies, to bound the fraction of used material and to impose a minimum length scale to the layout geometric features. Two separate finite element analyses are therefore required: the solution of the eigenvalue problem (Eq. (1)) for a number $n_{\omega}$ of modes, and a subsequent harmonic response solution (Eq. (4)), that is computed for $\omega_{h}=0.95 \cdot \omega_{s}$. The eigenvalue problem is solved for $n_{\omega}=25$ modes: this has proven to be a good choice since the drive and sense resonant frequencies keep among the computed $n_{\omega}$ ones for all the optimization steps in all the treated examples.

In Eq. 111), $\omega_{d}, \omega_{s}$ and $\omega_{s p}$ are the drive, sense and first spurious resonant frequencies, while $\omega_{d, d e s}$ and $\omega_{s, \text { des }}$ are the target drive and sense resonant frequencies. The constraints are set to match the desired target drive and sense resonant frequencies with a certain relative tolerance $\varepsilon$. Moreover, a $5 \%$ mismatch between the drive and the sense frequencies is imposed, while the first spurious mode is kept at least $40 \%$ higher than the sense frequency. Note that in this way the required ordering of the eigenfrequencies correspond to $\omega_{1}=\omega_{d}$, $\omega_{2}=\omega_{s}$ and $\omega_{3}=\omega_{s p}$. The definition of the different modes is based on the displacements of the center of gravity of the proof mass in the corresponding mode shapes: the drive mode is defined as the mode with a pure horizontal displacement of the proof mass, and the sense mode as the mode with a pure vertical displacement. The first spurious mode will be the one with the lowest frequency among the remaining modes.

A volume constraint is applied to set an upper bound $v^{*}$ to the amount of used material Vol, while geometric constraints allow to impose a minimum length-scale to the layout features and hence ensure manufacturability. In order to improve convergence to black and white layouts, the last constraints are imposed to explicitly penalize the use of grey elements. The formulation of geometric and grey penalization constraints will be discussed in Sections 4.4 and 4.5 respectively.

4.2 (P2) Lightweight suspending structure with target eigenfrequencies

In the formulation of the second optimization problem (P2) we eliminate the harmonic response and instead focus on matching the target resonant frequencies while minimizing the total mass. This leads to the following statement:

$$
\begin{array}{cl}
\min _{\gamma_{e}} & \mathrm{Vol}=\frac{\sum_{e} \overline{\tilde{\gamma}}_{e} v_{e}}{\sum_{e} v_{e}} \\
\text { subject to } & \frac{\left(\omega_{d}-\omega_{d, d e s}\right)^{2}}{\omega_{d, d e s}^{2}}-\varepsilon^{2} \leq 0 \\
& \frac{\left(\omega_{s}-\omega_{s, d e s}\right)^{2}}{\omega_{s, d e s}^{2}}-\varepsilon^{2} \leq 0 \\
& \frac{1.4 \cdot \omega_{s}}{\omega_{s p}}-1 \leq 0 \\
& \omega_{d, d e s}=0.95 \cdot \omega_{s, d e s} \\
& \left(-\omega_{i}^{2} \boldsymbol{M}+\boldsymbol{K}\right) \boldsymbol{\Phi}_{\boldsymbol{i}}=\mathbf{0}, i=1 \ldots n_{\omega}
\end{array}
$$

Geometric constraints

Grey penalization constraints

The objective function to be minimized is the amount of used material Vol. Besides leading to a lightweight suspending structure, this also helps the convergence of the algorithm to a feasible layout, avoiding undesired big portions of material attached to the suspending structure, that are associate to low-frequency local spurious modes.

The same constraints as in (P1) are imposed to match the target eigenfrequencies, to impose a minimum length-scale and to penalize grey areas.

(P2) requires to solve only an eigenvalue problem (Eq. (1)) per iteration, and the overall optimization process is therefore computationally cheaper than solving $(\mathrm{P} 1)$. However, since (P2) does not consider the harmonic response of the structure to the external angular rate, there is no way to guarantee that the sense response is maximized in the process. Our hypothesis is therefore that operating the MEMS gyroscope close to the sense resonant frequency should be adequate to ensure a high amplitude sense response.

4.3 (P3) Lightweight suspending structure with target eigenfrequencies, using static estimations

The formulation of the third topology optimization problem (P3) is similar to the one of (P2), but the solution of the eigenvalue problem in Eq. (1) is replaced by simple and computationally cheap static estimations.

The idea is to assume that low frequency modes are associated to rigid movements of the proof mass, with negligible internal dynamics of the suspending structure. In this way the mode shapes can be estimated as the static deformed shapes of the structure 


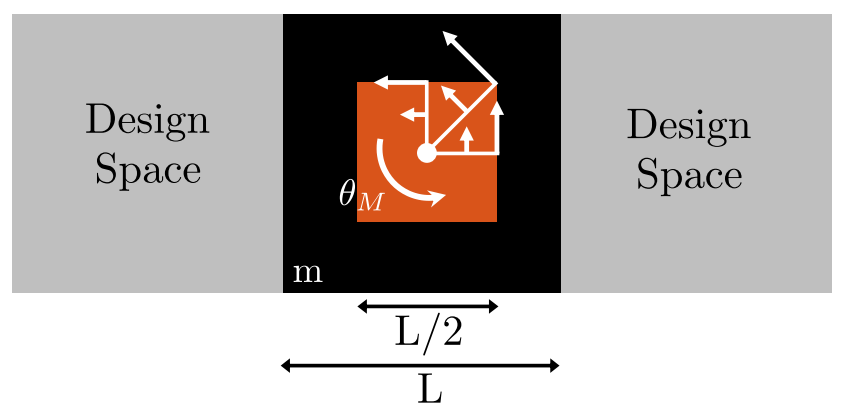

Fig. 5: Static estimation of the first spurious mode by imposing a static rotation $\theta_{M}$ to the proof mass.

when proper displacements of the proof mass are imposed. In particular, the drive mode shape can be estimated by imposing a horizontal static displacement $u_{x, M}$, whereas the sense mode shape can be estimated by imposing a static vertical displacement $u_{y, M}$ (cf. Fig. 3).

While the definition of the drive and sense mode shapes is straightforward, it can be difficult to predict which mode will be the first spurious one. The chosen guess is here to impose an in plane rotation $\theta_{M}$ to the proof mass. This is done by prescribing corresponding horizontal/vertical displacements to the nodes around its center of gravity. At this purpose, a square region with side equal to half the side of the proof mass (red area in Fig. 5 is considered: this dimension has proven to be a good trade off to avoid artificial localized effects while preserving the overall compliance of the structure. We note that it is possible to include several spurious modes to the static problem, but our choice has proven to be effective for the considered design cases.

Once the static deformation is computed, the frequency is estimated from the computation of the mass and the stiffness associated to the estimated mode shape.
The formulation of (P3) therefore becomes:

$$
\begin{array}{ll}
\min _{\gamma_{e}} & \operatorname{Vol}=\frac{\sum_{e} \overline{\tilde{\gamma}}_{e} v_{e}}{\sum_{e} v_{e}} \\
\text { subject to } & \frac{\left(\tilde{\omega}_{d}-\omega_{d, d e s}\right)^{2}}{\omega_{d, d e s}^{2}}-\varepsilon^{2} \leq 0 \\
& \frac{\left(\tilde{\omega}_{s}-\omega_{s, d e s}\right)^{2}}{\omega_{s, d e s}^{2}}-\varepsilon^{2} \leq 0 \\
& \frac{1.4 \cdot \tilde{\omega}_{s}}{\tilde{\omega}_{s p}}-1 \leq 0 \\
& \omega_{d, d e s}=0.95 \cdot \omega_{s, d e s} \\
& \tilde{\omega}_{i}=\sqrt{\frac{\tilde{k}_{m, i}}{\tilde{m}_{m, i}}}=\sqrt{\frac{\tilde{\boldsymbol{\Phi}}_{\boldsymbol{i}}{ }^{T} \boldsymbol{K} \tilde{\boldsymbol{\Phi}}_{\boldsymbol{i}}}{\tilde{\boldsymbol{\Phi}}_{\boldsymbol{i}}{ }^{T} \boldsymbol{M} \tilde{\boldsymbol{\Phi}}_{\boldsymbol{i}}}} \\
& \boldsymbol{K} \tilde{\boldsymbol{\Phi}}_{\boldsymbol{i}}=\boldsymbol{f}_{\boldsymbol{i}}, i=\mathrm{d}, \mathrm{s}, \mathrm{sp}
\end{array}
$$$$
\text { Geometric constraints }
$$

Grey penalization constraints

In Eq. (14) $\tilde{\omega}_{i}$ and $\tilde{\boldsymbol{\Phi}}_{\boldsymbol{i}}$ are the estimations of the eigenfrequencies and the eigenmodes, while $\tilde{k}_{m, i}$ and $\tilde{m}_{m, i}$ are the estimations of the modal stiffness and mass. The load vector $\boldsymbol{f}_{\boldsymbol{i}}$ contains the reaction forces in the static estimation of the $i$-th mode shape.

\subsection{Geometric constraints}

Geometric constraints are added to the presented design problems to impose a minimum length-scale for the optimized layouts, namely a minimum width for the geometric features.

In general, a length-scale is desirable both for manufacturability and for numerical considerations Poulsen, 2003. The structure is usually fabricated by etching or chemical deposition, and imposing a minimum lengthscale enhances the reliability of the fabrication process. From the numerical point of view, a minimum length scale means that the finest features of the design are not determined by the underlying finite element discretization. This gives invariance of the optimized layout under mesh refinement and the possibility to discretize the finest parts of the structure by a reasonable number of elements.

The formulation of geometric constraints follows the one proposed in Zhou et al., 2015]. The idea is inspired by the work on topology optimization with robust formulations Wang et al., 2011. Different realizations of the design are considered by changing the threshold used to compute the projected design fields: if $\eta$ gives the nominal design (in this case $\eta=0.5$ ), $\eta_{d}<\eta$ gives dilated designs and $\eta_{e}=1-\eta_{d}>\eta$ gives eroded designs 
Wang et al., 2011, Zhou et al., 2015. If all the designs in the range $\eta \in\left(\eta_{d}, \eta_{e}\right)$ share the same topology, the optimized nominal design possesses a minimum length scale. In particular, the imposed minimum length scale depends on the value $\eta_{d}$ for the dilated design: here $\eta_{d}=0.25$ and $\eta_{e}=1-\eta_{d}=0.75$ are considered, so that the minimum length scale corresponds to the filter radius $r_{\min }$ selected to compute the filtered densities Wang et al., 2011.

In order to formulate the geometric constraints, two structural indicator functions are defined to capture the inflection regions of the solid and void phases Zhou et al., 2015:

$I^{s}=\overline{\tilde{\gamma}} \cdot \exp \left(-c \cdot\|\nabla \tilde{\gamma}\|^{2}\right)$

$I^{v}=(1-\overline{\tilde{\gamma}}) \cdot \exp \left(-c \cdot\|\nabla \tilde{\gamma}\|^{2}\right)$

with: $c=r_{\min }^{4}$

where the subscripts $s$ and $v$ stand for the solid and void phases respectively, and the parameter $c$ of the exponential function is set to $c=r_{\text {min }}^{4}$ as suggested in Zhou et al., 2015.

Based on the indicator functions, the geometric constraints are imposed as:

$g^{s}=\frac{1}{n} \sum_{i \in \mathbb{N}} I_{i}^{s} \cdot\left[\min \left\{\left(\tilde{\gamma}_{i}-\eta_{e}\right), 0\right\}\right]^{2} \leq \varepsilon_{g c}$

$g^{v}=\frac{1}{n} \sum_{i \in \mathbb{N}} I_{i}^{v} \cdot\left[\min \left\{\left(\eta_{d}-\tilde{\gamma}_{i}\right), 0\right\}\right]^{2} \leq \varepsilon_{g c}$

where $n$ is the total number of elements in the discretization set $\mathbb{N}$, and $\varepsilon_{g c}$ is a given tolerance. Satisfying these two constraints allows to impose a minimum length scale.

\subsection{Grey penalization constraints}

Convergence to black and white layouts can be improved considering that, if grey connections are present in the structure, pushing grey variables towards void causes disconnections, and the eigenfrequencies approach zero. The use of grey connections can be therefore discouraged by bounding this change of eigenfrequencies.

It is therefore useful to define a new field of variables $\gamma_{e}^{(*)}$, in which intermediate design variables are pushed towards void. Considering the structure layout defined by the field $\gamma_{e}^{(*)}$, it is possible to compute the new drive and sense resonant frequencies $\omega_{d}^{(*)}$ and $\omega_{s}^{(*)}$. Their difference with respect to the original eigenfrequencies $\omega_{d}$ and $\omega_{s}$, related to the field $\overline{\tilde{\gamma}}_{e}$, will be then bound by grey penalization constraints. They can be formulated as:

$1-\frac{\omega_{d}^{(*)}}{\alpha_{\text {grey }} \cdot \omega_{d}} \leq 0$
$1-\frac{\omega_{s}^{(*)}}{\alpha_{\text {grey }} \cdot \omega_{s}} \leq 0$

Grey penalization constraints depend on the choice of the grey penalizing field $\gamma_{e}^{(*)}$ and on the lower bound parameter $0 \leq \alpha_{\text {grey }} \leq 1$. Some examples of possible definitions of these parameters in different design cases are provided in Sections 6 and 7

\subsection{Sensitivity Analysis}

The formulated optimization problems (P1), (P2) and (P3) (Eq. 11), 13), 14) can be solved for local optimality using a gradient-based optimizer such as the Method of Moving Asymptotes (MMA) by Svanberg Svanberg, 1987]. The evaluation of the sensitivities of the objective function and the constraints with respect to the design variables is therefore required.

In general, the sensitivities of the functions that depend only on the design field can be easily computed by analytically differentiating their expressions. On the other hand, the sensitivities of the functions that depend on the state of the system have to be computed exploiting the adjoint method when many design variables are considered. In the following the results of the adjoint sensitivity analysis for this latter type of functions are presented.

The gradient of a general function $\Psi$ with respect to the design field $\gamma_{e}$ is computed using the chain rule, considering the derivatives of the expressions in Eq. (7) and (9) of the filtered field $\tilde{\gamma}_{e}$ and of the projected field $\overline{\tilde{\gamma}}_{e}$ :

$\frac{\partial \Psi}{\partial \gamma_{e}}=\frac{\partial \Psi}{\partial \overline{\tilde{\gamma}}_{e}} \frac{\partial \overline{\tilde{\gamma}}_{e}}{\partial \tilde{\gamma}_{e}} \frac{\partial \tilde{\gamma}_{e}}{\partial \gamma_{e}}$

Concerning the harmonic response analysis (Eq. (4)) considered in $(\mathrm{P} 1)(\mathrm{Eq}, 11)$, the state and adjoint fields have been split into a real and an imaginary part to ensure well-defined derivatives. The sensitivities of the objective function of the first formulation with respect to the projected field are:

$\frac{\partial \Psi_{1}}{\partial \tilde{\tilde{\gamma}}_{e}}=\frac{1}{\psi_{1}^{(i t=0)}} \frac{\partial \psi_{1}}{\partial \tilde{\tilde{\gamma}}_{e}}$ 
The sensitivities of $\psi_{1}$ can be obtained as explained in Appendix A, namely:

$$
\begin{aligned}
\frac{\partial \psi_{1}}{\partial \tilde{\tilde{\gamma}}_{e}} & =\boldsymbol{\lambda}_{\mathbf{1}}^{T}\left(\frac{\partial \boldsymbol{K}}{\partial \overline{\tilde{\gamma}}_{e}}-\omega_{h}^{2} \frac{\partial \boldsymbol{M}}{\partial \overline{\tilde{\gamma}}_{e}}\right) \boldsymbol{u}_{\boldsymbol{h}, \boldsymbol{r}}+ \\
& -\omega_{h} \boldsymbol{\lambda}_{\mathbf{1}}^{T} \frac{\partial \boldsymbol{G}_{\boldsymbol{C}}\left(\Omega_{z}\right)}{\partial \overline{\tilde{\gamma}}_{e}} \boldsymbol{u}_{\boldsymbol{h}, \boldsymbol{i}}+ \\
& -\boldsymbol{\lambda}_{\mathbf{2}}^{T}\left(\frac{\partial \boldsymbol{K}}{\partial \overline{\tilde{\gamma}}_{e}}-\omega_{h}^{2} \frac{\partial \boldsymbol{M}}{\partial \overline{\tilde{\gamma}}_{e}}\right) \boldsymbol{u}_{\boldsymbol{h}, \boldsymbol{i}}+ \\
& -\omega_{h} \boldsymbol{\lambda}_{\mathbf{2}}{ }^{T} \frac{\partial \boldsymbol{G}_{\boldsymbol{C}}\left(\Omega_{z}\right)}{\partial \overline{\tilde{\gamma}}_{e}} \boldsymbol{u}_{\boldsymbol{h}, \boldsymbol{r}}+ \\
& +\boldsymbol{\lambda}_{\mathbf{3}}{ }^{T}\left(\frac{\partial \boldsymbol{K}}{\partial \overline{\tilde{\gamma}}_{e}}-\omega_{s}^{2} \frac{\partial \boldsymbol{M}}{\partial \tilde{\tilde{\gamma}}_{e}}\right) \boldsymbol{\Phi}_{\boldsymbol{s}}+ \\
& +\lambda_{4} \boldsymbol{\Phi}_{\boldsymbol{s}}{ }^{T} \frac{\partial \boldsymbol{M}}{\partial \overline{\tilde{\gamma}}_{e}} \boldsymbol{\Phi}_{\boldsymbol{s}}
\end{aligned}
$$

where $\boldsymbol{\Phi}_{\boldsymbol{s}}$ is the sense mode shape, $\boldsymbol{u}_{\boldsymbol{h}, \boldsymbol{r}}$ and $\boldsymbol{u}_{\boldsymbol{h}, \boldsymbol{i}}$ indicate the real and imaginary parts of vector $\boldsymbol{u}_{\boldsymbol{h}}$ and $\left(\boldsymbol{\lambda}_{\mathbf{1}}, \boldsymbol{\lambda}_{\mathbf{2}}\right)$ are computed from the following adjoint problem:

$$
\begin{aligned}
& \boldsymbol{\lambda}_{\mathbf{1}}=\operatorname{Re}\left(\boldsymbol{\lambda}^{*}\right), \boldsymbol{\lambda}_{\mathbf{2}}=\operatorname{Im}\left(\boldsymbol{\lambda}^{*}\right) \\
& \left(-\omega_{h}^{2} \boldsymbol{M}+i \omega_{h} \boldsymbol{G}_{\boldsymbol{C}}\left(\Omega_{z}\right)^{T}+\boldsymbol{K}\right) \boldsymbol{\lambda}^{*}=-(\boldsymbol{a}-i \boldsymbol{b})
\end{aligned}
$$

with:

$\boldsymbol{a}=\left(\frac{\partial \psi_{1}}{\partial\left(\boldsymbol{u}_{\boldsymbol{h}, \boldsymbol{r}}\right)}\right)^{T}$ and $\quad \boldsymbol{b}=\left(\frac{\partial \psi_{1}}{\partial\left(\boldsymbol{u}_{\boldsymbol{h}, \boldsymbol{i}}\right)}\right)^{T}$

The other two adjoint vectors $\left(\boldsymbol{\lambda}_{\mathbf{3}}, \lambda_{4}\right)$ are given by the solution of the following adjoint problem that depends on the solution of Eq. (21):

$\left[\begin{array}{cc}\boldsymbol{K}-\omega_{s}^{2} \boldsymbol{M} & 2 \boldsymbol{M} \boldsymbol{\Phi}_{\boldsymbol{s}} \\ 2 \omega_{s} \boldsymbol{\Phi}_{\boldsymbol{s}}^{T} \boldsymbol{M} & 0\end{array}\right]\left[\begin{array}{c}\boldsymbol{\lambda}_{\mathbf{3}} \\ \lambda_{4}\end{array}\right]=\left[\begin{array}{l}0 \\ p\end{array}\right]$

with the right hand side given as

$$
\begin{aligned}
p= & -2 \alpha^{2} \omega_{s} \boldsymbol{u}_{\boldsymbol{h}, \boldsymbol{r}}{ }^{T} \boldsymbol{M} \boldsymbol{\lambda}_{\mathbf{1}}+ \\
& -\alpha \boldsymbol{u}_{\boldsymbol{h}, \boldsymbol{i}}{ }^{T} \boldsymbol{G}_{\boldsymbol{C}}\left(\Omega_{z}\right)^{T} \boldsymbol{\lambda}_{\mathbf{1}}+ \\
& +2 \alpha^{2} \omega_{s} \boldsymbol{u}_{\boldsymbol{h}, \boldsymbol{i}}{ }^{T} \boldsymbol{M} \boldsymbol{\lambda}_{\mathbf{2}}+ \\
& -\alpha \boldsymbol{u}_{\boldsymbol{h}, \boldsymbol{r}}{ }^{T} \boldsymbol{G}_{\boldsymbol{C}}\left(\Omega_{z}\right)^{T} \boldsymbol{\lambda}_{\mathbf{2}}
\end{aligned}
$$

Hence, we remark that Eq. 21 must be solved before Eq. (23).
The sensitivities of the constraints on the eigenfrequencies with respect to the projected field can be computed as:

$$
\begin{aligned}
& \frac{\partial}{\partial \overline{\tilde{\gamma}}_{e}}\left(\frac{\left(\omega_{d}-\omega_{d, d e s}\right)^{2}}{\omega_{d, d e s}^{2}}-\varepsilon^{2}\right)=\frac{2\left(\omega_{d}-\omega_{d, d e s}\right)}{\omega_{d, d e s}^{2}} \frac{\partial \omega_{d}}{\partial \overline{\tilde{\gamma}}_{e}} \\
& \frac{\partial}{\partial \overline{\tilde{\gamma}}_{e}}\left(\frac{\left(\omega_{s}-\omega_{s, d e s}\right)^{2}}{\omega_{s, d e s}^{2}}-\varepsilon^{2}\right)=\frac{2\left(\omega_{s}-\omega_{s, d e s}\right)}{\omega_{s, d e s}^{2}} \frac{\partial \omega_{s}}{\partial \tilde{\tilde{\gamma}}_{e}} \\
& \frac{\partial}{\partial \overline{\tilde{\gamma}}_{e}}\left(\frac{1.4 \cdot \omega_{s}}{\omega_{s p}}-1\right)=\frac{1.4}{\omega_{s p}^{2}}\left(\frac{\partial \omega_{s}}{\partial \overline{\tilde{\gamma}}_{e}} \omega_{s p}-\frac{\partial \omega_{s p}}{\partial \tilde{\tilde{\gamma}}_{e}} \omega_{s}\right)
\end{aligned}
$$

Exploiting the expressions for the sensitivities of the $i$-th eigenvalue $\omega_{i}^{2}$ Bendsoe and Sigmund, 2004, the gradient of the $i$-th eigenfrequency with respect to the projected field is:

$\frac{\partial \omega_{i}}{\partial \tilde{\tilde{\gamma}}_{e}}=\frac{1}{2 \omega_{i}} \boldsymbol{\Phi}_{i}{ }^{T}\left(\frac{\partial \boldsymbol{K}}{\partial \tilde{\tilde{\gamma}}_{e}}-\omega_{i}^{2} \frac{\partial \boldsymbol{M}}{\partial \overline{\tilde{\gamma}}_{e}}\right) \boldsymbol{\Phi}_{\boldsymbol{i}}$

assuming that $\boldsymbol{\Phi}_{\boldsymbol{i}}$ is mass normalized. Note the no adjoint is needed for Eq. 26.

The sensitivities of the static estimation of resonant frequencies can be computed as:

$\frac{\partial \tilde{\omega}_{i}}{\partial \tilde{\tilde{\gamma}}_{e}}=\frac{1}{2 \tilde{\omega}_{i} \tilde{m}_{m, i}^{2}}\left(\frac{\partial \tilde{k}_{m, i}}{\partial \tilde{\tilde{\gamma}}_{e}} \tilde{m}_{m, i}-\frac{\partial \tilde{m}_{m, i}}{\partial \tilde{\tilde{\gamma}}_{e}} \tilde{k}_{m, i}\right)$

where the sensitivities of the statically estimated modal stiffness and mass can be obtained as explained in Appendix B and are:

$\frac{\partial \tilde{k}_{m, i}}{\partial \tilde{\tilde{\gamma}}_{e}}=\tilde{\boldsymbol{\Phi}}_{\boldsymbol{i}}{ }^{T} \frac{\partial \boldsymbol{K}}{\partial \tilde{\tilde{\gamma}}_{e}} \tilde{\boldsymbol{\Phi}}_{\boldsymbol{i}}$
$\frac{\partial \tilde{m}_{m, i}}{\partial \tilde{\tilde{\gamma}}_{e}}=\tilde{\boldsymbol{\Phi}}_{\boldsymbol{i}}^{T} \frac{\partial \boldsymbol{M}}{\partial \overline{\tilde{\gamma}}_{e}} \tilde{\boldsymbol{\Phi}}_{\boldsymbol{i}}+\boldsymbol{\mu}^{T} \boldsymbol{K} \tilde{\boldsymbol{\Phi}}_{\boldsymbol{i}}$

with $\boldsymbol{\mu}$ being the solution of the following adjoint problem:

$\left[\begin{array}{cc}\boldsymbol{K}_{f f} & \boldsymbol{K}_{\boldsymbol{f c}} \\ \mathbf{0} & \boldsymbol{I}\end{array}\right] \boldsymbol{\mu}=\left[\begin{array}{c}-2 \boldsymbol{M}_{\boldsymbol{f}} \tilde{\boldsymbol{\Phi}}_{\boldsymbol{i}} \\ \mathbf{0}\end{array}\right]$

In Eq. 29) $\boldsymbol{K}_{\boldsymbol{f} \boldsymbol{f}}$ is a selection of the rows and columns of the matrix $\boldsymbol{K}$ corresponding to the free degrees of freedom, $\boldsymbol{K}_{\boldsymbol{f c}}$ is a selection of the rows corresponding to the free degrees of freedom and of the columns corresponding to constrained degrees of freedom and $\boldsymbol{M}_{\boldsymbol{f}}$ is a selection of the rows of the matrix $\boldsymbol{M}$ corresponding to the free degrees of freedom. 
For what concerns the sensitivities of the drive and sense frequencies $\omega_{d}^{(*)}$ and $\omega_{s}^{(*)}$, corresponding to the field $\gamma_{e}^{(*)}$, the expressions in Eq. (26) and (27) still apply, provided that the derivatives with respect to the projected field $\frac{\partial}{\partial \tilde{\bar{\gamma}}_{e}}$ are substituted with the derivatives with respect to the grey penalizing field $\frac{\partial}{\partial \gamma_{e}^{(*)}}$.

We note that the presented sensitivity computations have been verified by finite differences.

\section{Details of the optimization algorithm}

The scheme of the proposed optimization algorithm is presented in Fig. 6.

First of all, the structure is initialized: the material properties are defined, the design space is discretized by finite elements, boundary conditions are assigned and the design variables are initialized to an intermediate value $\gamma_{e}=0.4$. This value has been chosen based on numerical experiments and have proven to be a good choice. However, we remind that the design problems treated are all non-convex and hence that the initial distribution will have an impact on the final results.

At each optimization step, the filtering and projection schemes are applied and the needed finite element analyses are performed. The obtained results are used to compute the values of the objective function and the constraints of the optimization problem, together with the computation of the geometric constraints and grey penalization constraints.

Next, the sensitivities of the objective function and the constraints are computed, and the Method of Moving Asymptotes (MMA) Svanberg, 1987] is used as the mathematical programming method that provides an updated design variables field. The finite element analyses followed by the sensitivity analysis are performed again on this new structure to obtain a new updated design variables field by MMA. The process is repeated until the maximum difference between consecutive design variables fields is lower than a certain tolerance Bendsoe and Sigmund, 2004, here set to $10^{-3}$.

\section{Numerical experiments: solution of the different optimization problems for a benchmark design case}

In this Section the different formulated optimization problems (P1), (P2) and (P3) (Eq. (11), (13), (14)) are solved for the benchmark design case in Fig. 7, and the results are discussed and compared.

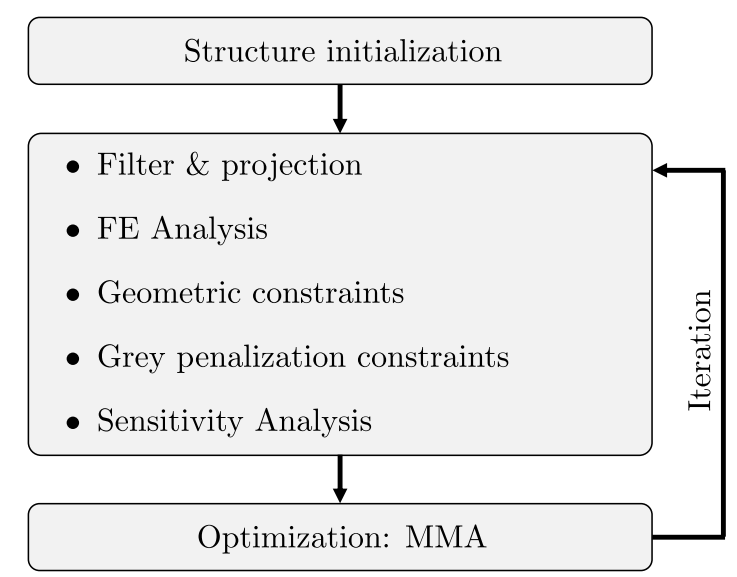

Fig. 6: Scheme of the proposed topology optimization algorithm.

\subsection{Setup of the design space}

Referring to Fig. 7, the aim is here to design the suspending structure on the two sides of a square proof mass with size $128 \times 128$. On each side, an available space with size $158 \times 128$ is set for the design of the suspending structure. The whole area is discretized by finite elements with dimensions $1 \times 1$ and symmetries are imposed to the structure with respect to the vertical and horizontal axes. This follows typical industrial choices: non symmetrical topologies can in fact easily couple the ideal horizontal/vertical drive/sense motions of the structure, increasing the quadrature error of the sensor and degrading its performance Acar and Shkel, 2009. Since we consider ideally decoupled drive and sense modes, we remark that the maximization of the sensor performance proposed in problem (P1) only focuses on the response to the external angular rate, and no minimization of the offset nor of the quadrature error of the gyroscope has been considered.

The proof mass is described by a fixed "passive" solid domain: no design variables are assigned to the corresponding area, that remains solid in the optimization process. A void padding of dimension $d_{e x t}$ is included along all the borders of the design domain as in Clausen and Andreassen, 2017, in order to avoid undesired boundary effects on the subsequent filters and geometric constraints. As suggested in Clausen and Andreassen, 2017], $d_{e x t}$ is set to be equal to the filter radius $r_{\text {min }}$.

The location of the connection between the proof mass and the design domain for the suspending structure is assigned by small passive solid portion with dimensions $2 d_{e x t} \times 2 d_{e x t}$, that is attached to the proof mass and crosses the void padding of the design domain. 


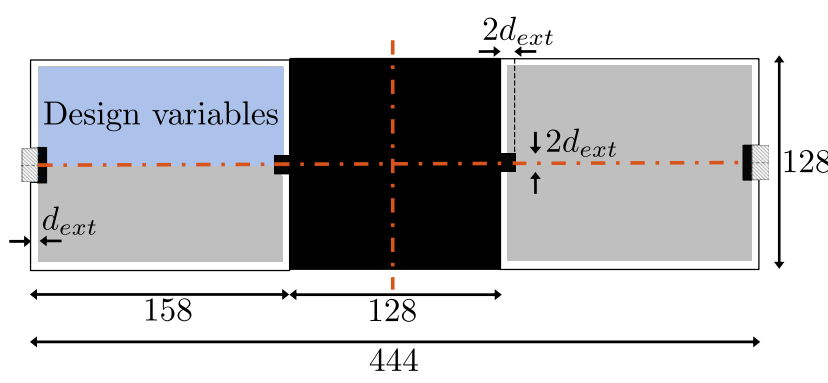

Fig. 7: Setup of the design space for the benchmark design case.

By way of example, in this case we have considered a unitary out-of-plane thickness of the structure, a unitary Young modulus $E_{0}=1$, a Poisson ratio $\nu=0.3$ and a density $\rho_{0}=1.76 \times 10^{-10}$.

\subsection{Optimization parameters}

The layout of the suspension structure is optimized in the design space using a filter radius $r_{\min }=4$, that also corresponds to the imposed length-scale as explained in Section 4.4

For the present design case, a soft penalization of grey is employed. In particular, grey penalization constraints (Section 4.5) are imposed using a lower bound parameter $\alpha_{\text {grey }}=0.35$ and an eroded field of variables for $\gamma_{e}^{(*)}$, that is computed projecting the filtered field with a threshold parameter $\eta_{e}=0.75$. Thus:

$\gamma_{e}^{(*)}=\frac{\tanh \left(\beta \eta_{e}\right)+\tanh \left(\beta\left(\tilde{\gamma}_{e}-\eta_{e}\right)\right)}{\tanh \left(\beta \eta_{e}\right)+\tanh \left(\beta\left(1-\eta_{e}\right)\right)}$

A continuation scheme is applied to the sharpness parameter $\beta$ of the projection scheme and to the tolerances $\varepsilon$ and $\varepsilon_{g c}$, to gradually enforce a black and white layout that satisfies the constraints with the desired tolerances. In particular, the desired tolerance $\varepsilon$ for the constraints on the target frequencies is set to $3 \cdot 10^{-4}$, while the tolerance $\varepsilon_{g c}$ for the geometric constraints is decreased during the optimization as a function of the projection parameter $\beta$ (Fig. 8).

Starting from $\beta=1$, the projection parameter is kept constant for 50 iterations and then increased by a factor 1.2 when the constraints are satisfied with a tolerance of $2 \cdot 10^{-4}$. When $\beta$ changes, constraints are slightly relaxed increasing the tolerances $\varepsilon$ and $\varepsilon_{g c}$ by a factor of 100 and decreasing them back to their desired values through 13 iterations. The process is then repeated increasing $\beta$ each time by a factor of 1.2 up to $\beta=64$. Also, for the presented design examples we have used the following MMA parameters:

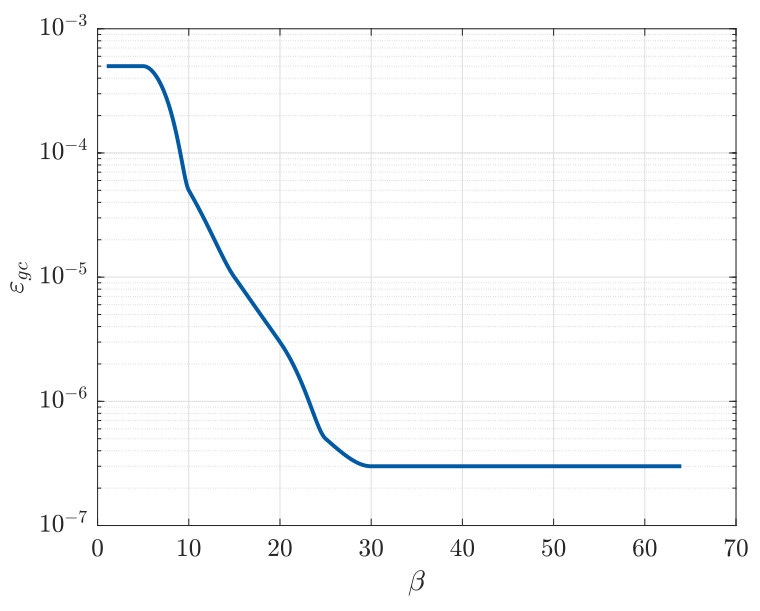

Fig. 8: Continuation scheme on the constraint tolerance $\varepsilon_{g c}$ as a function of the projection parameter $\beta$.

$a_{0}=1, a_{i}=0, c_{i}=1000, d_{i}=0$, whose nomenclature and explanation are provided in Svanberg, 2002]. We note that the optimization parameters have been chosen based on exhaustive numerical experiments.

As underlined in Zhou et al., 2015, the application of geometric constraints is effective after an initial topology has formed. For this reason, they are introduced later into the topology optimization process, for $\beta>7$, and the same applies for the grey penalization constraints.

\subsection{Optimized layouts}

Fig. 9 shows the different optimized layouts. As reported in Table 1, the suspension structure is optimized for target drive and sense frequencies equal to $3.8 \mathrm{~Hz}$ and $4 \mathrm{~Hz}$ respectively. Layout (1a), (1b), (1c) are optimized solving problem (P1), considering a test external angular $\Omega_{z}=10^{3} \cdot \omega_{s, \text { des }}$ in the dynamic analysis and using different available volume fractions $v^{*}$. Instead, layouts (1d) and (1e) correspond to the solution of (P2) and (P3) respectively. It can be also seen how the different layouts are conceptually similar, with a rhomboidal part providing the required compliance and a stiffer part close to the anchored area.

In Table 2 and Table 3 a quantitative comparison between the performance of the different layouts is presented, with focus on the resonant frequencies of the structure, the response to the external angular rate, the final volume fraction and the computational cost of the associated design problem. These results form the basis for the discussion of the three proposed design formulations which will be treated next. 
(1a)

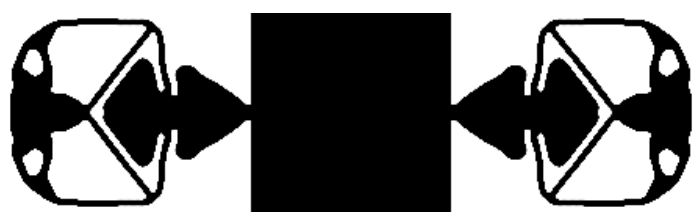

(1c)

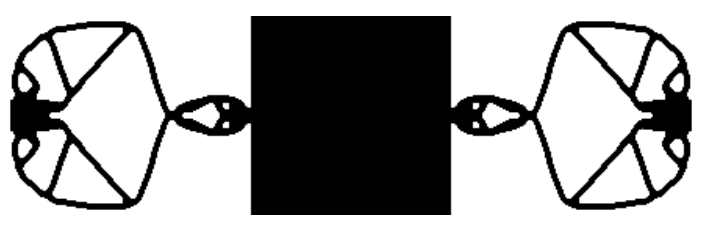

(1b)

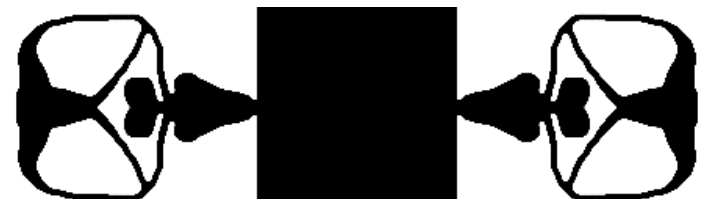

(1d)

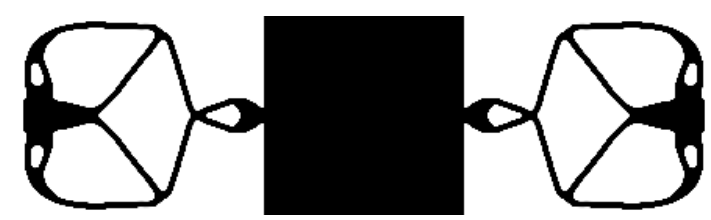

(1e)

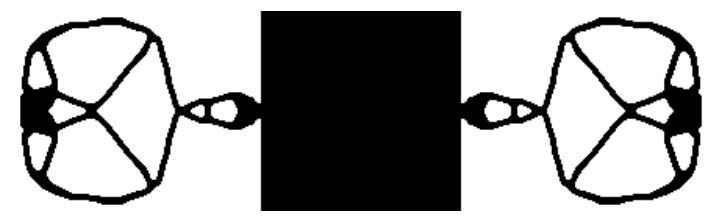

Fig. 9: Different optimized layouts for the benchmark design case, corresponding to the optimization parameters reported in Table 1 .

(a)

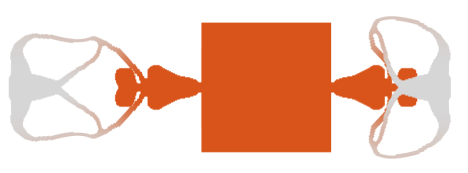

(b)

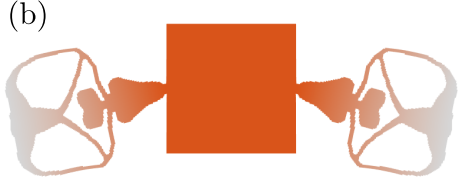

(c)

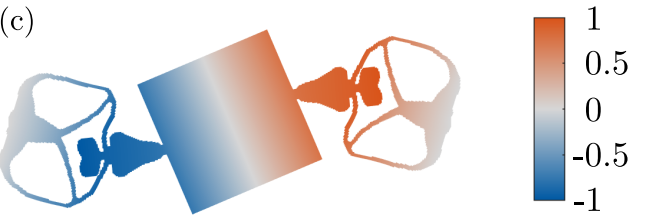

Fig. 10: Mode shapes of Layout (1b) shown in Fig. 9. (a) First mode shape (drive - frequency $3.8008 \mathrm{~Hz}, U_{x}$ contour plot). (b) Second mode shape (sense - frequency $3.9970 \mathrm{~Hz}, U_{y}$ contour plot). (c) Third mode shape (spurious frequency $7.7764 \mathrm{~Hz}, U_{y}$ contour plot).

(a)

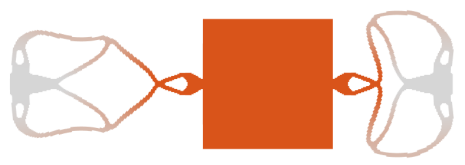

(b)

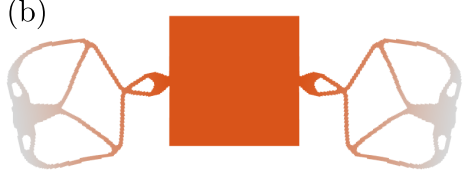

(c)

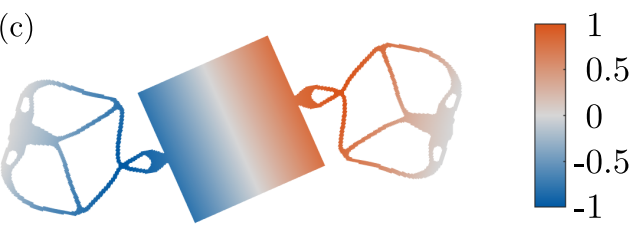

Fig. 11: Mode shapes of Layout (1d) shown in Fig. 9. (a) First mode shape (drive - frequency $3.7991 \mathrm{~Hz}, U_{x}$ contour plot). (b) Second mode shape (sense - frequency $3.9990 \mathrm{~Hz}, U_{y}$ contour plot). (c) Third mode shape (spurious frequency $7.5871 \mathrm{~Hz}, U_{y}$ contour plot).

(a)

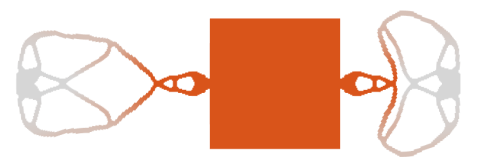

(b)

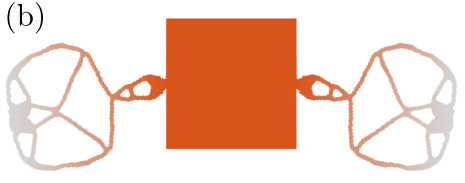

(c)

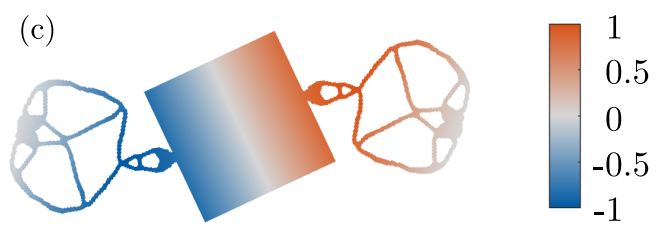

Fig. 12: Mode shapes of Layout (1e) shown in Fig. 9. (a) First mode shape (drive - frequency $3.7994 \mathrm{~Hz}, U_{x}$ contour plot). (b) Second mode shape (sense - frequency $3.9900 \mathrm{~Hz}, U_{y}$ contour plot). (c) Third mode shape (spurious frequency $8.0080 \mathrm{~Hz}, U_{y}$ contour plot). 


\begin{tabular}{|c|c|c|c|c|}
\hline Layout & Problem & $\boldsymbol{v}^{\boldsymbol{*}}$ & $\boldsymbol{f}_{\boldsymbol{d}, \boldsymbol{d e s}}$ & $\boldsymbol{f}_{\boldsymbol{s}, \boldsymbol{d e s}}$ \\
\hline (1a) & $(\mathrm{P} 1)$ & 1 & $3.8 \mathrm{~Hz}$ & $4 \mathrm{~Hz}$ \\
\hline (1b) & $(\mathrm{P} 1)$ & 0.5 & $3.8 \mathrm{~Hz}$ & $4 \mathrm{~Hz}$ \\
\hline (1c) & $(\mathrm{P} 1)$ & 0.4217 & $3.8 \mathrm{~Hz}$ & $4 \mathrm{~Hz}$ \\
\hline (1d) & $(\mathrm{P} 2)$ & - & $3.8 \mathrm{~Hz}$ & $4 \mathrm{~Hz}$ \\
\hline (1e) & $(\mathrm{P} 3)$ & - & $3.8 \mathrm{~Hz}$ & $4 \mathrm{~Hz}$ \\
\hline
\end{tabular}

Table 1: Benchmark design case: optimization parameters for the different layouts shown in Fig. 9.

\subsection{Resonant frequencies}

Table 2 lists the values of the resonant frequencies corresponding to the different layouts, while Fig. 10, 11 and 12 show the drive, sense and first spurious mode shapes of the layouts (1b), (1d) and (1e). In all cases the target values for the drive and sense frequencies are matched, and the first spurious frequency is maintained sufficiently higher than the sense one.

Table 2 also shows the validity of the static estimation of the resonant frequencies used in the third formulation. An almost perfect match with the results of modal analysis is achieved for the drive and sense frequencies, while a small error $(2.66 \%)$ is associated to the estimation of the first spurious frequency. This is likely due to the fact that the internal dynamics of the elastic parts start playing a role at the first spurious frequency. Despite the small introduced error, the accuracy of the static estimation of the resonant frequencies is here enough to match the target drive and sense frequencies in the optimization process, and to keep the first spurious frequency sufficiently higher than the sense one.

\subsection{Response to the external angular rate}

A comparison between the different optimized layout in terms of the objective function $\Psi_{1}$ of $(\mathrm{P} 1)$ is shown in Table 3. when an external angular $\Omega_{z}=10^{3} \cdot \omega_{s, \text { des }}$ is considered. In addition, Fig. 13 and 14 show the trend of $\psi_{1}=\left\|\frac{u_{y, M}}{u_{x, M}}\right\|$ with respect to the excitation frequency, for the different optimized layouts. This quantity represents the the modulus of the transfer function between the imposed drive displacement $u_{x, M}$ and the response $u_{y, M}$ along the sense axis.

As expected, the highest dynamic response to the external angular rate is achieved when (P1) is solved (layouts (1a), (1b) and (1c)), and the final dynamic response increases with the available volume fraction.

Refer to Fig. 9 and consider for example layout (1b) and (1d): it can be seen how the higher available volume fraction of layout (1b) is exploited in the optimization

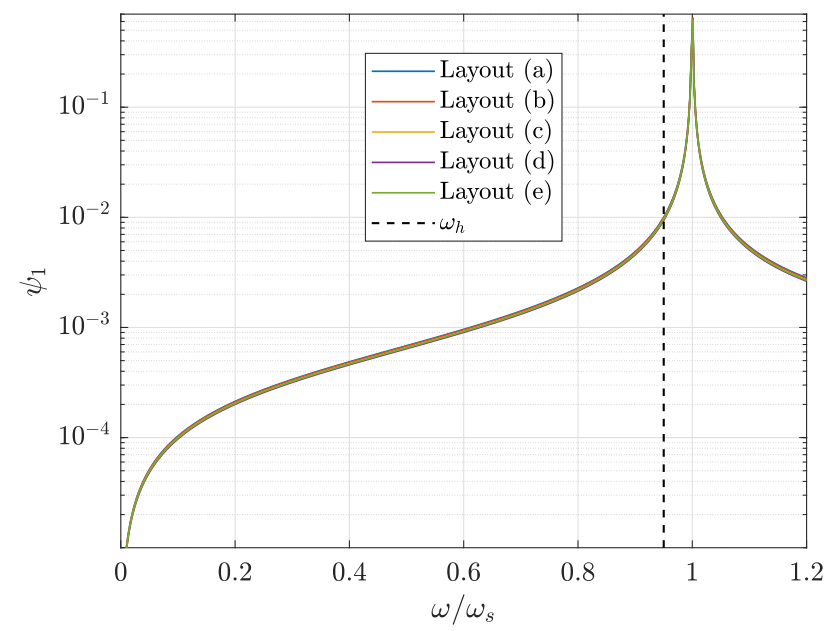

Fig. 13: Benchmark design case: trend of $\psi_{1}=\left\|\frac{u_{y, M}}{u_{x, M}}\right\|$ with respect to the excitation frequency, for the different optimized layouts.

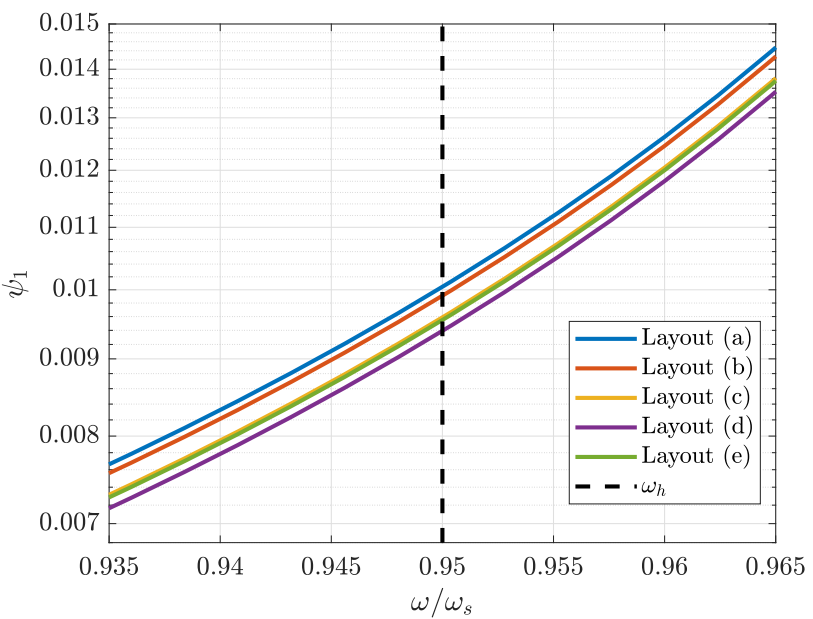

Fig. 14: Benchmark design case: detail of the trend of $\psi_{1}=\left\|\frac{u_{y, M}}{u_{x, M}}\right\|$ shown in Fig. 13 around the excitation frequency $\omega_{h}=0.95 \cdot \omega_{s}$.

process to concentrate material close to the proof mass. Referring to Fig. 10, this additional portion of mass has the same displacement of the proof mass in the drive motion, but only a fraction of the displacement of the proof mass in the sense motion. This allows to have a bigger total mass participating to the drive motion, maximizing the total generated Coriolis force, and to have a smaller total mass in the sense motion, increasing the response to the Coriolis force along the sense direction.

The concentration of material close to the proof mass is marginal, but still effective, for layout (1c), where a maximum volume fraction equal to the one obtained in layout $(1 \mathrm{~d})$ is imposed. The effect is instead 


\begin{tabular}{|c|c|c|c|c|c|c|c|}
\hline Layout & Problem & $\boldsymbol{f}_{\boldsymbol{d}}$ & $\boldsymbol{f}_{\boldsymbol{s}}$ & $\boldsymbol{f}_{\boldsymbol{s p}}$ & $\tilde{\boldsymbol{f}}_{\boldsymbol{d}}$ & $\tilde{\boldsymbol{f}}_{\boldsymbol{s}}$ & $\tilde{\boldsymbol{f}}_{\boldsymbol{s} \boldsymbol{p}}$ \\
\hline$(1 \mathrm{a})$ & $(\mathrm{P} 1)$ & $3.8007 \mathrm{~Hz}$ & $3.9975 \mathrm{~Hz}$ & $6.8114 \mathrm{~Hz}$ & - & - & - \\
\hline$(1 \mathrm{~b})$ & $(\mathrm{P} 1)$ & $3.8008 \mathrm{~Hz}$ & $3.9970 \mathrm{~Hz}$ & $7.7764 \mathrm{~Hz}$ & - & - & - \\
\hline$(1 \mathrm{c})$ & $(\mathrm{P} 1)$ & $3.8010 \mathrm{~Hz}$ & $3.9942 \mathrm{~Hz}$ & $7.9971 \mathrm{~Hz}$ & - & - & - \\
\hline$(1 \mathrm{~d})$ & $(\mathrm{P} 2)$ & $3.7991 \mathrm{~Hz}$ & $3.9990 \mathrm{~Hz}$ & $7.5871 \mathrm{~Hz}$ & - & - & - \\
\hline$(1 \mathrm{e})$ & $(\mathrm{P} 3)$ & $3.7994 \mathrm{~Hz}$ & $3.9900 \mathrm{~Hz}$ & $8.0080 \mathrm{~Hz}$ & $3.8000 \mathrm{~Hz}$ & $3.9989 \mathrm{~Hz}$ & $8.2212 \mathrm{~Hz}$ \\
\hline
\end{tabular}

Table 2: Resonant frequencies of the different optimized layouts shown in Fig. 9 .

\begin{tabular}{|c|c|c|c|c|c|c|}
\hline Layout & Problem & $\boldsymbol{\Psi}_{\mathbf{1}}$ & $\begin{array}{c}\text { Max. error on } \\
\text { freq. estimation }\end{array}$ & Vol & $\begin{array}{c}\text { Computational cost } \\
\text { (normalized) }\end{array}$ & N iterations \\
\hline$(1 \mathrm{a})$ & $(\mathrm{P} 1)$ & 0.6500 & $0 \%$ & 0.5583 & $100 \%$ & 1270 \\
\hline$(1 \mathrm{~b})$ & $(\mathrm{P} 1)$ & 0.6326 & $0 \%$ & 0.5000 & $100 \%$ & 1441 \\
\hline$(1 \mathrm{c})$ & $(\mathrm{P} 1)$ & 0.5921 & $0 \%$ & 0.4217 & $100 \%$ & 1703 \\
\hline$(1 \mathrm{~d})$ & $(\mathrm{P} 2)$ & 0.5683 & $0 \%$ & 0.4217 & $56.2 \%$ & 1232 \\
\hline$(1 \mathrm{e})$ & $(\mathrm{P} 3)$ & 0.5874 & $2.66 \%$ & 0.4158 & $20 \%$ & 1411 \\
\hline
\end{tabular}

Table 3: Benchmark design case: comparison of the different optimized layouts (Fig. 9] in terms of final performance and computational cost of the associated optimization problem.

maximized in layout (1a), where there is no constraint on the maximum available volume fraction and a big portion of mass is placed to increase the dynamic response.

\subsection{Computational cost}

Table 3 shows also a comparison between the computational costs of the different formulations, that are normalized with respect to the computational cost of (P1).

While the different layouts are obtained with comparable numbers of iterations, it can be seen how (P1) is the most expensive design problem in terms of time per iteration, since it requires both the modal and the harmonic response analyses with the associated adjoint solutions.

Instead, (P3) brings a good advantage by replacing the modal analysis with a static estimation of the resonant frequencies: the computational time is reduced by almost a factor of 3 with respect to (P2).

\section{Numerical experiments: application to a real MEMS gyroscope design case}

In Section 6 the different optimization problems have been presented and compared for a benchmark design case. In this Section, we choose (P3) and apply it to a real MEMS gyroscope design case, considering typical dimensions and material properties of a real world structure. Static estimations of resonant frequencies are in fact associated with a low computational cost, and

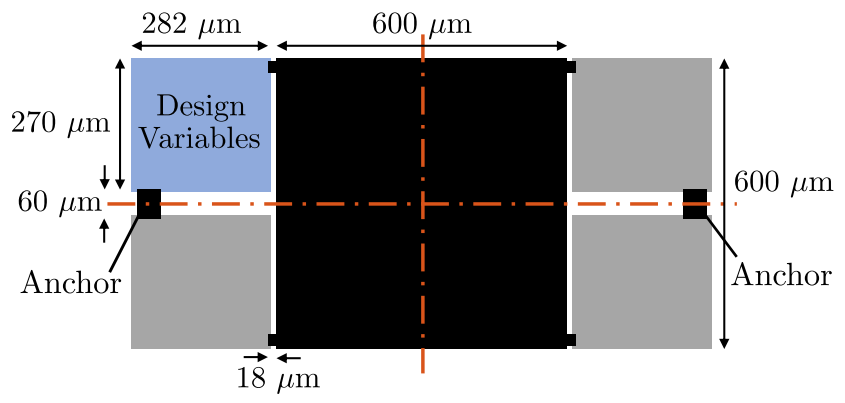

Fig. 15: Setup of the design space for the real MEMS gyroscope design case.

are shown to be accurate enough to allow the optimizer to match the target resonant frequencies.

7.1 Setup of the design space and optimization parameters

Fig. 15 shows the set up of the real world gyroscope design problem. It includes a proof mass with dimensions $600 \mu \mathrm{m} \times 600 \mu \mathrm{m}$, and we enforce the symmetry of the suspension structure as shown with the blue coloring of the design domain. The proof mass and the suspending structure are separated by a passive void strip similar to the design case presented in Section 6 . and their connection is imposed through a small passive solid portion located close to the corner of the proof mass. The anchor points are imposed as passive solid areas. The out-of-plane thickness of the structure is set to $25 \mu \mathrm{m}$, and the material properties of polysilicon have been considered, i.e. a Young modulus of 
$E_{0}=148000 \mathrm{MPa}$, a Poisson ratio of $\nu=0.23$ and a mass density of $\rho_{0}=2330 \mathrm{~kg} / \mathrm{m}^{3}$.

The design domain is discretized by a mesh of 320000 finite elements, each with dimensions $1.5 \mu \mathrm{m} \times 1.5 \mu \mathrm{m}$. A filter radius of $r_{\text {min }}=4$ is imposed which leads to a length-scale of $6 \mu \mathrm{m}$ for the minimum allowable size of the geometric features. In this way the imposed minimum length-scale follows typical dimensions of compliant elements in MEMS gyroscope structures Acar and Shkel, 2009, and the finest geometric features are discretized by at least 4 elements.

With respect to the benchmark case, in the real world gyroscope design case the optimization algorithm has shown to be more prone to exploit grey connections to match the target resonant frequencies. Therefore, we employ a harder grey penalization, defining the field $\gamma_{e}^{(*)}$ with an additional projection applied on the field $\overline{\tilde{\gamma}}_{e}$ (Eq. (99). Thus:

$\gamma_{e}^{(*)}=\frac{\tanh \left(\beta_{2} \eta_{2}\right)+\tanh \left(\beta_{2}\left(\overline{\tilde{\gamma}}_{e}-\eta_{2}\right)\right)}{\tanh \left(\beta_{2} \eta_{2}\right)+\tanh \left(\beta_{2}\left(1-\eta_{2}\right)\right)}$

The new threshold and sharpness parameter have been set to $\eta_{2}=1$ and $\beta_{2}=3 \beta$ respectively. Fig. 16 plots the new projection scheme for several values of $\beta_{2}$. Again, the lower bound parameter is set to $\alpha_{\text {grey }}=$ 0.35 .

The formulated grey penalization constraints are active from the beginning of the optimization process, while geometric constraints are considered only for $\beta>$ 7. The same procedure explained in Section 6.2 is followed for the continuation schemes on the sharpness parameter $\beta$ (and consequently to $\beta_{2}$ ) and on the constraints tolerances $\varepsilon$ and $\varepsilon_{g c}$.

In the following we summarize the differences in the optimization parameters between the benchmark and the real word design cases:

$\mathrm{i}$ in the real world design case we define the grey penalization field using an additional projection, opposed to the eroded design with simple projection used in the benchmark case

ii in the real world design case we invoke grey penalization constraints from the beginning of the optimization, compared to when $\beta$ exceeds 7 in the benchmark case

\subsection{Optimization results}

Fig. 17 shows the different optimized layouts for the real MEMS gyroscope design case and the corresponding data for each example design are shown in Table 4 and 5 .

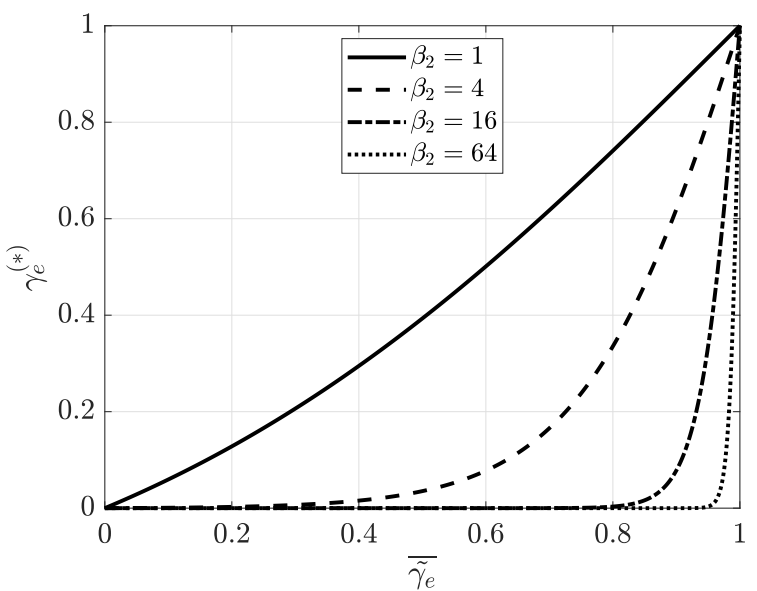

Fig. 16: Additional projection scheme defining the grey penalizing field in the real MEMS gyroscope design case (Eq. (31)), for threshold $\eta_{2}=1$ and different sharpness parameters $\beta_{2}$.

The optimization problem is initially solved for $\omega_{s, \text { des }}=$ $40 \mathrm{kHz}$, investigating the behaviour of the algorithm when grey penalization is first not considered (Fig. 17 (2a)) and then introduced (Fig. $17(2 b)$ ). Results show that, when grey penalization is not applied, disconnections appear in the support structure and the design has no physical significance (layout (2a)). Convergence to a connected and feasible layout is instead achieved when grey penalization is introduced (layout (2b)).

The behaviour of the algorithm is then tested when the target sense resonant frequency is decreased, when still applying grey penalization. Layouts (2c) and (2d) in Fig. 17 show the results optimizing for $\omega_{s, \text { des }}=$ $32 \mathrm{kHz}$ and $\omega_{s, \text { des }}=24 \mathrm{kHz}$. In these cases the target eigenfrequencies are matched by employing an increasing amount of meandering in the layout, in order to design a suspending structure that has a progressively increasing compliance. The mode shapes of layout (2b), (2c) and (2d) are shown in Fig. 18,19 and 20 respectively.

Finally, Fig. 17 (2e) shows that for a further decrease of the target sense resonant frequency to $\omega_{s, d e s}=$ $20 \mathrm{kHz}$ convergence is not achieved. Although the structure contains evidence and outlines of an intricate meandering layout, the algorithm is not able to satisfy all the constraint and gets stuck at $\beta=2.99$ (cf. Table 4). 

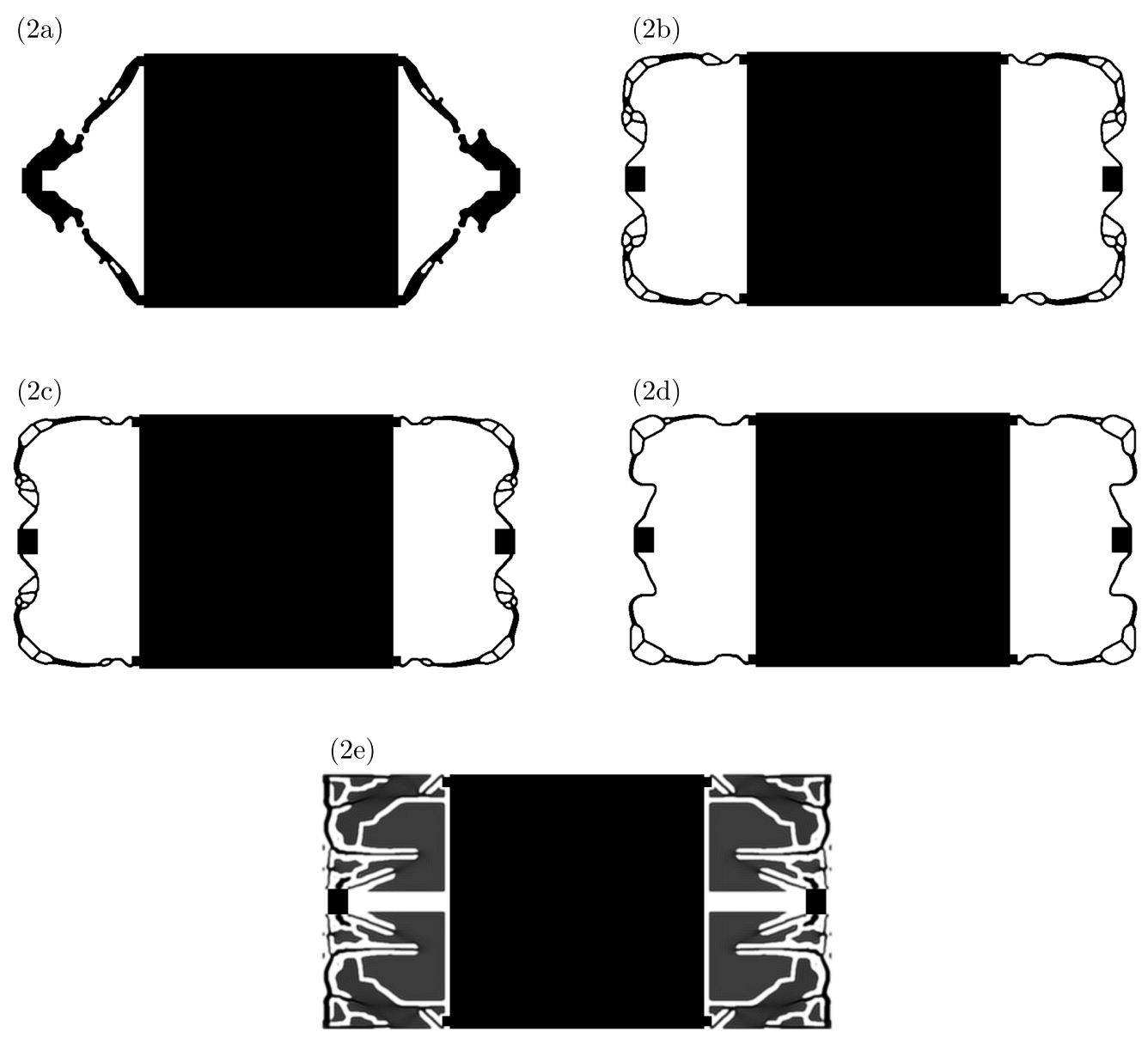

Fig. 17: Different optimized layouts for the real MEMS gyroscope design case, corresponding to the optimization parameters reported in Table 4.

\begin{tabular}{|c|c|c|c|c|}
\hline Layout & Problem & $\boldsymbol{f}_{\boldsymbol{d}, \boldsymbol{d e s}}$ & $\boldsymbol{f}_{\boldsymbol{s}, \boldsymbol{d e s}}$ & Grey penalization \\
\hline$(2 \mathrm{a})$ & $(\mathrm{P} 3)$ & $38000 \mathrm{~Hz}$ & $40000 \mathrm{~Hz}$ & No \\
\hline$(2 \mathrm{~b})$ & $(\mathrm{P} 3)$ & $38000 \mathrm{~Hz}$ & $40000 \mathrm{~Hz}$ & Yes \\
\hline$(2 \mathrm{c})$ & $(\mathrm{P} 3)$ & $30400 \mathrm{~Hz}$ & $32000 \mathrm{~Hz}$ & Yes \\
\hline$(2 \mathrm{~d})$ & $(\mathrm{P} 3)$ & $22800 \mathrm{~Hz}$ & $24000 \mathrm{~Hz}$ & Yes \\
\hline$(2 \mathrm{e})$ & $(\mathrm{P} 3)$ & $19000 \mathrm{~Hz}$ & $20000 \mathrm{~Hz}$ & Yes \\
\hline
\end{tabular}

Table 4: Real MEMS gyroscope design case: optimization parameters for the different layouts shown in Fig. 17

\begin{tabular}{|c|c|c|c|c|c|c|c|c|}
\hline Layout & Vol & $\boldsymbol{f}_{\boldsymbol{d}}$ & $\boldsymbol{f}_{\boldsymbol{s}}$ & $\boldsymbol{f}_{\boldsymbol{s} \boldsymbol{p}}$ & $\tilde{\boldsymbol{f}}_{\boldsymbol{d}}$ & $\tilde{\boldsymbol{f}}_{\boldsymbol{s}}$ & $\tilde{\boldsymbol{f}}_{\boldsymbol{s} \boldsymbol{p}}$ & Physical sound \\
\hline$(2 \mathrm{a})$ & 0.553 & $36814 \mathrm{~Hz}$ & $38902 \mathrm{~Hz}$ & $75421 \mathrm{~Hz}$ & $36815 \mathrm{~Hz}$ & $38905 \mathrm{~Hz}$ & $75421 \mathrm{~Hz}$ & No \\
\hline$(2 \mathrm{~b})$ & 0.532 & $37989 \mathrm{~Hz}$ & $39994 \mathrm{~Hz}$ & $97107 \mathrm{~Hz}$ & $37992 \mathrm{~Hz}$ & $39996 \mathrm{~Hz}$ & $97127 \mathrm{~Hz}$ & Yes \\
\hline$(2 \mathrm{c})$ & 0.530 & $30393 \mathrm{~Hz}$ & $31995 \mathrm{~Hz}$ & $78818 \mathrm{~Hz}$ & $30394 \mathrm{~Hz}$ & $31996 \mathrm{~Hz}$ & $78825 \mathrm{~Hz}$ & Yes \\
\hline$(2 \mathrm{~d})$ & 0.523 & $22794 \mathrm{~Hz}$ & $23993 \mathrm{~Hz}$ & $61146 \mathrm{~Hz}$ & $22797 \mathrm{~Hz}$ & $23999 \mathrm{~Hz}$ & $61163 \mathrm{~Hz}$ & Yes \\
\hline$(2 \mathrm{e})$ & 0.743 & $18576 \mathrm{~Hz}$ & $18979 \mathrm{~Hz}$ & $28822 \mathrm{~Hz}$ & $18886 \mathrm{~Hz}$ & $19089 \mathrm{~Hz}$ & $30864 \mathrm{~Hz}$ & No: stop at $\beta=2.99$ \\
\hline
\end{tabular}

Table 5: Real MEMS gyroscope design case: final material usage, resonant frequencies and physical soundness for the different optimized layouts shown in Fig. 17. 
(a)

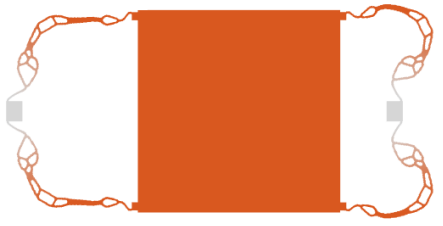

(b)

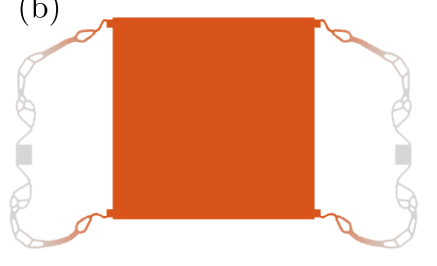

(c)

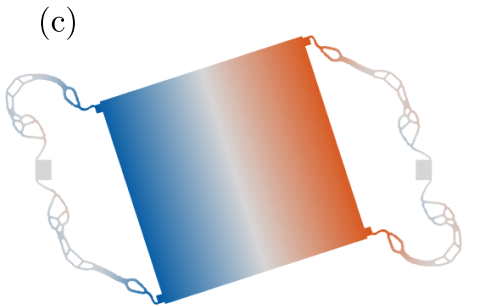

1

0.5

0

$-0.5$

Fig. 18: Mode shapes of Layout (2b) shown in Fig. 17. (a) First mode shape (drive - frequency $37989 \mathrm{~Hz}, U_{x}$ contour plot). (b) Second mode shape (sense - frequency $39994 \mathrm{~Hz}, U_{y}$ contour plot). (c) Third mode shape (spurious frequency $97107 \mathrm{~Hz}, U_{y}$ contour plot).

(a)

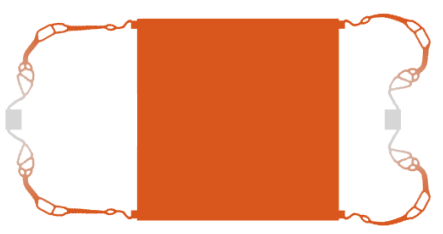

(b)

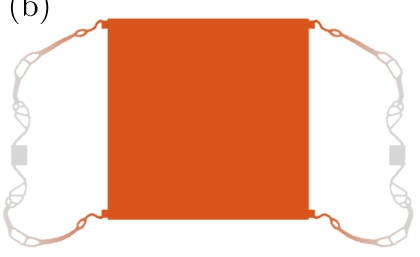

(c)

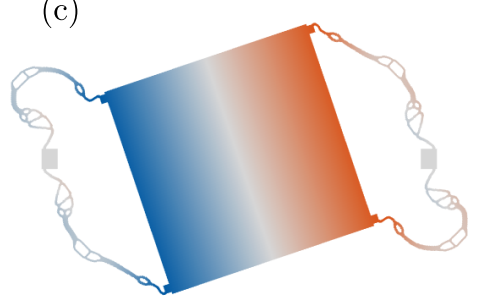

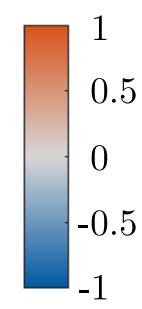

Fig. 19: Mode shapes of Layout (2c) shown in Fig. 17. (a) First mode shape (drive - frequency $30393 \mathrm{~Hz}, U_{x}$ contour plot). (b) Second mode shape (sense - frequency $31995 \mathrm{~Hz}, U_{y}$ contour plot). (c) Third mode shape (spurious frequency $78818 \mathrm{~Hz}, U_{y}$ contour plot).

(a)

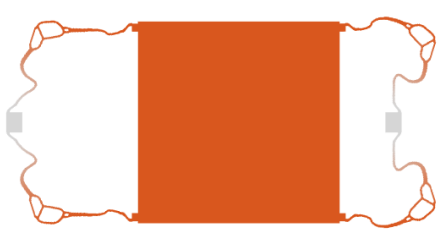

(b)

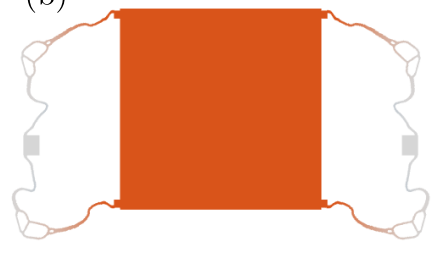

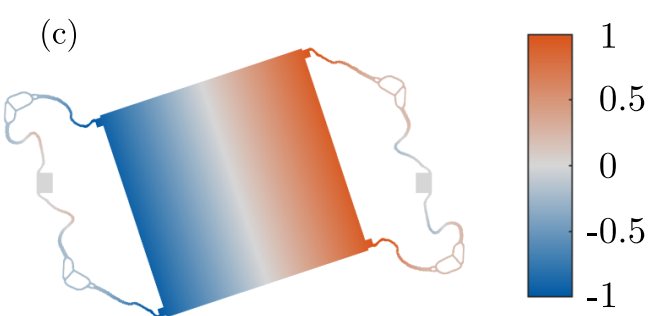

Fig. 20: Mode shapes of Layout (2d) shown in Fig. 17. (a) First mode shape (drive - frequency $22794 \mathrm{~Hz}, U_{x}$ contour plot). (b) Second mode shape (sense - frequency $23993 \mathrm{~Hz}, U_{y}$ contour plot). (c) Third mode shape (spurious frequency $61146 \mathrm{~Hz}, U_{y}$ contour plot). 


\section{Conclusions}

In this paper the topology optimization approach has been applied to the design of MEMS gyroscopes, considering an in plane, single mass layout.

Three different problem formulations with varying computational complexity have been presented and compared for a benchmark design case. Results show how the approach allows to satisfy the typical design requirements of the MEMS gyroscope structure. The desired ordering of the resonant frequencies is achieved: the target drive and the sense resonant frequencies are matched, while spurious modes are kept far enough from the operation range. The sensor response to the angular rate can be maximized if included in the formulation, though a proper distribution of mass in the layout. Manufacturability can be ensured by imposing a minimum length-scale to the geometric features through geometric constraints. In order to improve convergence to black and white layouts, constraints can be included in the formulations to employ an explicit penalization of grey elements, that have proved its effectiveness in several presented numerical experiments.

A formulation that exploits static estimations of the resonant frequencies has been also presented: it leads to a substantial reduction of the computational cost with respect to the full modal analysis, resulting only in a small estimation error on the resonant frequencies of interest.

This last formulation has been applied to a real MEMS gyroscope design case, for which the behaviour of the algorithm has been tested for decreasing target resonant frequencies. It has been seen how an increasing meandering is exploited by the algorithm in order to progressively increase the compliance of the suspending structure. This applies down to a certain target frequency, below which convergence has not been guaranteed.

Future research work will focus on the extension of the proposed approach to real MEMS gyroscopes design cases, considering also the out-of-plane motion of the structure, using e.g. shell finite elements, and more complex layouts with several proof mass and sense axes. In addition, different formulations will be investigated to design gyroscope layouts with even lower resonant frequencies.

\section{Replication of the results}

The manuscript provides all the information needed by readers to replicate the presented results. The authors have stored all data related to the presented examples and ensured that all relevant parameters are stated clearly throughout the manuscript. Furthermore, the code can be made available upon request to the corresponding author.

Acknowledgements Daniele Giannini and Francesco Braghin would like to thank the AMG R\&D team of STMicroelectronics, who provided the industrial insight and expertise that greatly assisted the research. Niels Aage acknowledges the support of the Villum Foundation as part of the InnoTop VILLUM Investigator project.

Funding information This study was funded by STMicroelectronics (grant code 081/16CR) and Villum Foundation (InnoTop VILLUM Investigator project).

Conflict of interest Francesco Braghin and Daniele Giannini received research grants from the commercial company STMicroelectronics, active in the MEMS field.

\section{Appendix A: adjoint sensitivity analysis of the} sense response

In order to compute the sensitivities of the sense response $\psi_{1}$ (Eq. 12) with respect to the projected field, we first consider the corresponding harmonic response problem:

$\left(-\alpha^{2} \omega_{s}^{2} \boldsymbol{M}+i \alpha \omega_{s} \boldsymbol{G}_{\boldsymbol{C}}\left(\Omega_{z}\right)+\boldsymbol{K}\right) \boldsymbol{u}_{\boldsymbol{h}}=\boldsymbol{f}_{\boldsymbol{h}}$

and rewrite it introducing the dynamic stiffness matrix $\boldsymbol{S}$ as well as expliciting the dependencies on the projected field and on the sense resonant frequency:

$\boldsymbol{S}\left(\overline{\tilde{\gamma}}_{e}, \omega_{s}\right) \boldsymbol{u}_{\boldsymbol{h}}=\boldsymbol{f}_{\boldsymbol{h}}$

Considering that:

$\boldsymbol{S}=\boldsymbol{S}_{\boldsymbol{r}}+i \boldsymbol{S}_{\boldsymbol{i}}$

$\boldsymbol{u}_{\boldsymbol{h}}=\boldsymbol{u}_{\boldsymbol{h}, \boldsymbol{r}}+i \boldsymbol{u}_{\boldsymbol{h}, \boldsymbol{i}}$

$\boldsymbol{f}_{\boldsymbol{h}}=\boldsymbol{f}_{\boldsymbol{h}, \boldsymbol{r}}+i \boldsymbol{f}_{\boldsymbol{h}, \boldsymbol{i}}$

Eq. (33) can be split into its real and imaginary parts as:

$S_{r} u_{h, r}-S_{i} u_{h, i}=f_{h, r}$

$S_{r} u_{h, i}+S_{i} u_{h, r}=f_{h, i}$

Referring to Eq. (12), the sense response $\psi_{1}$ can be expressed as a function of the projected field and of the real and imaginary parts of the harmonic displacements vector:

$\psi_{1}=\psi_{1}\left(\overline{\tilde{\gamma}}_{e}, \boldsymbol{u}_{\boldsymbol{h}, \boldsymbol{r}}, \boldsymbol{u}_{\boldsymbol{h}, \boldsymbol{i}}\right)$ 
As usual for adjoint sensitivity analysis, we add to this function extra (vanishing) terms, that are related to the harmonic response, to the sense eigenvalue problem and to the normalization of the sense modal shape:

$$
\begin{aligned}
\widehat{\psi}_{1}= & \psi_{1}+\boldsymbol{\lambda}_{\mathbf{1}}^{T}\left(\boldsymbol{S}_{\boldsymbol{r}} \boldsymbol{u}_{\boldsymbol{h}, \boldsymbol{r}}-\boldsymbol{S}_{\boldsymbol{i}} \boldsymbol{u}_{\boldsymbol{h}, \boldsymbol{i}}-\boldsymbol{f}_{\boldsymbol{h}, \boldsymbol{r}}\right)+ \\
& -\boldsymbol{\lambda}_{\mathbf{2}}{ }^{T}\left(\boldsymbol{S}_{\boldsymbol{r}} \boldsymbol{u}_{\boldsymbol{h}, \boldsymbol{i}}+\boldsymbol{S}_{\boldsymbol{i}} \boldsymbol{u}_{\boldsymbol{h}, \boldsymbol{r}}-\boldsymbol{f}_{\boldsymbol{h}, \boldsymbol{i}}\right)+ \\
& +\boldsymbol{\lambda}_{\mathbf{3}}{ }^{T}\left(\boldsymbol{K}-\omega_{s}^{2} \boldsymbol{M}\right) \boldsymbol{\Phi}_{\boldsymbol{s}}+\lambda_{4}\left(\boldsymbol{\Phi}_{\boldsymbol{s}}^{T} \boldsymbol{M} \boldsymbol{\Phi}_{\boldsymbol{s}}-1\right)
\end{aligned}
$$

We now compute the sensitivities of the above function with respect to the projected field, that after rearranging the terms become:

$$
\begin{aligned}
& \frac{\partial \widehat{\psi}_{1}}{\partial \overline{\tilde{\gamma}}_{e}}=\boldsymbol{\lambda}_{\mathbf{1}}{ }^{T} \frac{\partial \boldsymbol{S}_{\boldsymbol{r}}}{\partial \overline{\tilde{\gamma}}_{e}} \boldsymbol{u}_{\boldsymbol{h}, \boldsymbol{r}}-\boldsymbol{\lambda}_{\mathbf{1}}{ }^{T} \frac{\partial \boldsymbol{S}_{\boldsymbol{i}}}{\partial \overline{\tilde{\gamma}}_{e}} \boldsymbol{u}_{\boldsymbol{h}, \boldsymbol{i}}+ \\
& -\boldsymbol{\lambda}_{\mathbf{2}}{ }^{T} \frac{\partial \boldsymbol{S}_{\boldsymbol{r}}}{\partial \tilde{\tilde{\gamma}}_{e}} \boldsymbol{u}_{\boldsymbol{h}, \boldsymbol{i}}-\boldsymbol{\lambda}_{\mathbf{2}}{ }^{T} \frac{\partial \boldsymbol{S}_{\boldsymbol{i}}}{\partial \tilde{\tilde{\gamma}}_{e}} \boldsymbol{u}_{\boldsymbol{h}, \boldsymbol{r}}+ \\
& +\boldsymbol{\lambda}_{\mathbf{3}}{ }^{T}\left(\frac{\partial \boldsymbol{K}}{\partial \overline{\tilde{\gamma}}_{e}}-\omega_{s}^{2} \frac{\partial \boldsymbol{M}}{\partial \overline{\tilde{\gamma}}_{e}}\right) \boldsymbol{\Phi}_{\boldsymbol{s}}+ \\
& +\lambda_{4} \boldsymbol{\Phi}_{\boldsymbol{s}}{ }^{T} \frac{\partial \boldsymbol{M}}{\partial \tilde{\tilde{\gamma}}_{e}} \boldsymbol{\Phi}_{\boldsymbol{s}}+ \\
& +\left(\frac{\partial \psi_{1}}{\partial \boldsymbol{u}_{\boldsymbol{h}, \boldsymbol{r}}}+\boldsymbol{\lambda}_{\mathbf{1}}{ }^{T} \boldsymbol{S}_{\boldsymbol{r}}-\boldsymbol{\lambda}_{\mathbf{2}}{ }^{T} \boldsymbol{S}_{\boldsymbol{i}}\right) \frac{\partial \boldsymbol{u}_{\boldsymbol{h}, \boldsymbol{r}}}{\partial \overline{\tilde{\gamma}}_{e}}+ \\
& +\left(\frac{\partial \psi_{1}}{\partial \boldsymbol{u}_{\boldsymbol{h}, \boldsymbol{i}}}-\boldsymbol{\lambda}_{\mathbf{1}}{ }^{T} \boldsymbol{S}_{\boldsymbol{i}}-\boldsymbol{\lambda}_{\mathbf{2}}{ }^{T} \boldsymbol{S}_{\boldsymbol{r}}\right) \frac{\partial \boldsymbol{u}_{\boldsymbol{h}, \boldsymbol{i}}}{\partial \overline{\tilde{\gamma}}_{e}}+ \\
& +\left(\boldsymbol{\lambda}_{\mathbf{1}}{ }^{T} \frac{\partial \boldsymbol{S}_{\boldsymbol{r}}}{\partial \omega_{s}} \boldsymbol{u}_{\boldsymbol{h}, \boldsymbol{r}}-\boldsymbol{\lambda}_{\mathbf{1}}{ }^{T} \frac{\partial \boldsymbol{S}_{\boldsymbol{i}}}{\partial \omega_{s}} \boldsymbol{u}_{\boldsymbol{h}, \boldsymbol{i}}+\right. \\
& -\boldsymbol{\lambda}_{\mathbf{2}}{ }^{T} \frac{\partial \boldsymbol{S}_{\boldsymbol{r}}}{\partial \omega_{s}} \boldsymbol{u}_{\boldsymbol{h}, \boldsymbol{i}}-\boldsymbol{\lambda}_{\mathbf{2}}{ }^{T} \frac{\partial \boldsymbol{S}_{\boldsymbol{i}}}{\partial \omega_{s}} \boldsymbol{u}_{\boldsymbol{h}, \boldsymbol{r}}+ \\
& \left.-2 \omega_{s} \boldsymbol{\lambda}_{\mathbf{3}}{ }^{T} \boldsymbol{M} \boldsymbol{\Phi}_{\boldsymbol{s}}\right) \frac{\partial \omega_{s}}{\partial \tilde{\tilde{\gamma}}_{e}}+ \\
& +\left(\boldsymbol{\lambda}_{\mathbf{3}}^{T}\left(\boldsymbol{K}-\omega_{s}^{2} \boldsymbol{M}\right)+\lambda_{4} 2 \boldsymbol{\Phi}_{\boldsymbol{s}}^{T} \boldsymbol{M}\right) \frac{\partial \boldsymbol{\Phi}_{\boldsymbol{s}}}{\partial \tilde{\tilde{\gamma}}_{e}}
\end{aligned}
$$

In order to eliminate the expressions involving $\frac{\partial \boldsymbol{u}_{\boldsymbol{h}, \boldsymbol{r}}}{\partial \overline{\tilde{\gamma}}_{e}}$ and $\frac{\partial \boldsymbol{u}_{\boldsymbol{h}, \boldsymbol{i}}}{\partial \overline{\tilde{\gamma}}_{e}}$, we require the first two parenthesis to vanish:

$\boldsymbol{\lambda}_{\mathbf{1}}^{T} \boldsymbol{S}_{\boldsymbol{r}}-\boldsymbol{\lambda}_{\mathbf{2}}{ }^{T} \boldsymbol{S}_{\boldsymbol{i}}=-\frac{\partial \psi_{1}}{\partial \boldsymbol{u}_{\boldsymbol{h}, \boldsymbol{r}}}$

$\boldsymbol{\lambda}_{\mathbf{1}}^{T} \boldsymbol{S}_{\boldsymbol{i}}+\boldsymbol{\lambda}_{\mathbf{2}}^{T} \boldsymbol{S}_{\boldsymbol{r}}=\frac{\partial \psi_{1}}{\partial \boldsymbol{u}_{\boldsymbol{h}, \boldsymbol{i}}}$

Multiplying the second equation in 39 by $i$ and adding it to the first one yields:

$$
\left(\boldsymbol{\lambda}_{\mathbf{1}}^{T}+i \boldsymbol{\lambda}_{\mathbf{2}}^{T}\right)\left(\boldsymbol{S}_{\boldsymbol{r}}+i \boldsymbol{S}_{\boldsymbol{i}}\right)=-\left(\frac{\partial \psi_{1}}{\partial \boldsymbol{u}_{\boldsymbol{h}, \boldsymbol{r}}}-i \frac{\partial \psi_{1}}{\partial \boldsymbol{u}_{\boldsymbol{h}, \boldsymbol{i}}}\right)
$$

that after introducing $\boldsymbol{\lambda}^{*}=\boldsymbol{\lambda}_{\mathbf{1}}+i \boldsymbol{\lambda}_{\mathbf{2}}$ and $\boldsymbol{S}=$ $\boldsymbol{S}_{\boldsymbol{r}}+i \boldsymbol{S}_{\boldsymbol{i}}$, and after transposing, gives Eq. 21 that allows to compute the adjoints $\boldsymbol{\lambda}_{\mathbf{1}}$ and $\boldsymbol{\lambda}_{\mathbf{2}}$.

In order to compute the adjoints $\boldsymbol{\lambda}_{\mathbf{3}}$ and $\lambda_{4}$, we instead eliminate the expressions involving $\frac{\partial \omega_{s}}{\partial \tilde{\bar{\gamma}}_{e}}$ and $\frac{\partial \boldsymbol{\Phi}_{s}}{\partial \tilde{\bar{\gamma}}_{e}}$, i.e. we require the last two parenthesis in Eq. (38) to vanish. After transposing, this yields Eq. 23) that allows to compute $\boldsymbol{\lambda}_{\mathbf{3}}$ and $\lambda_{4}$.

The final expression of the sensitivities of $\psi_{1}$ (Eq. (20) ) can be obtained from Eq. (38) by removing the four eliminated terms.

\section{Appendix B: adjoint sensitivity analysis of the statically estimated modal stiffness and mass}

The sensitivities of the statically estimated resonant frequencies (Eq. (27) ) are obtained from the sensitivities of the statically estimated modal stiffness and mass (Eq. (28).

In order to compute the sensitivities of the statically estimated modal stiffness $\tilde{k}_{m, i}$ with respect to the projected field, we first consider the correspondent static load case and partition it into the sets of free and constrained degrees of freedom (pedices $f$ and $c$ respectively):

$$
\left[\begin{array}{ll}
K_{f f} & K_{f c} \\
K_{c f} & K_{c c}
\end{array}\right]\left[\begin{array}{c}
\tilde{\Phi}_{i, f} \\
\tilde{\Phi}_{i, c}
\end{array}\right]=\left[\begin{array}{l}
f_{i, f} \\
f_{i, c}
\end{array}\right]=\left[\begin{array}{c}
0 \\
f_{i, c}
\end{array}\right]
$$

Following the partition, the expression for $\tilde{k}_{m, i}$ becomes:

$$
\begin{aligned}
\tilde{k}_{m, i}= & \tilde{\boldsymbol{\Phi}}_{\boldsymbol{i}, \boldsymbol{f}}{ }^{T} \boldsymbol{K}_{\boldsymbol{f} \boldsymbol{f}} \tilde{\boldsymbol{\Phi}}_{\boldsymbol{i}, \boldsymbol{f}}+2 \tilde{\boldsymbol{\Phi}}_{\boldsymbol{i}, \boldsymbol{c}}^{T} \boldsymbol{K}_{\boldsymbol{c f} \boldsymbol{f}} \tilde{\boldsymbol{\Phi}}_{\boldsymbol{i}, \boldsymbol{f}}+ \\
& +\tilde{\boldsymbol{\Phi}}_{\boldsymbol{i}, \boldsymbol{c}}{ }^{T} \boldsymbol{K}_{\boldsymbol{c c}} \tilde{\boldsymbol{\Phi}}_{\boldsymbol{i}, \boldsymbol{c}}
\end{aligned}
$$

We then add to $\tilde{k}_{m, i}$ two vanishing terms related to the two sets of equations in Eq. (41):

$$
\begin{aligned}
\widehat{\tilde{k}}_{m, i}= & \tilde{\boldsymbol{\Phi}}_{\boldsymbol{i}, \boldsymbol{f}}{ }^{T} \boldsymbol{K}_{\boldsymbol{f} \boldsymbol{f}} \tilde{\boldsymbol{\Phi}}_{\boldsymbol{i}, \boldsymbol{f}}+2 \tilde{\boldsymbol{\Phi}}_{\boldsymbol{i}, \boldsymbol{c}}{ }^{T} \boldsymbol{K}_{\boldsymbol{c f}} \tilde{\boldsymbol{\Phi}}_{\boldsymbol{i}, \boldsymbol{f}}+ \\
& +\tilde{\boldsymbol{\Phi}}_{\boldsymbol{i}, \boldsymbol{c}}{ }^{T} \boldsymbol{K}_{\boldsymbol{c c}} \tilde{\boldsymbol{\Phi}}_{\boldsymbol{i}, \boldsymbol{c}}+\boldsymbol{\nu}_{\mathbf{1}}{ }^{T}\left(\boldsymbol{K}_{\boldsymbol{f} \boldsymbol{f}} \tilde{\boldsymbol{\Phi}}_{\boldsymbol{i}, \boldsymbol{f}}+\right. \\
& \left.+\boldsymbol{K}_{\boldsymbol{f c}} \tilde{\boldsymbol{\Phi}}_{\boldsymbol{i}, \boldsymbol{c}}\right)+\boldsymbol{\nu}_{\mathbf{2}}{ }^{T}\left(\boldsymbol{K}_{\boldsymbol{c f}} \tilde{\boldsymbol{\Phi}}_{\boldsymbol{i}, \boldsymbol{f}}+\right. \\
& \left.+\boldsymbol{K}_{\boldsymbol{c c}} \tilde{\boldsymbol{\Phi}}_{\boldsymbol{i}, \boldsymbol{c}}-\boldsymbol{f}_{\boldsymbol{i}, \boldsymbol{c}}\right)
\end{aligned}
$$


The sensitivities of the above function with respect to the projected field, after rearranging the terms, become:

$$
\begin{aligned}
& \frac{\partial \widehat{\tilde{k}}_{m, i}}{\partial \tilde{\tilde{\gamma}}_{e}}=\tilde{\boldsymbol{\Phi}}_{\boldsymbol{i}, \boldsymbol{f}}{ }^{T} \frac{\partial \boldsymbol{K}_{\boldsymbol{f} \boldsymbol{f}}}{\partial \tilde{\tilde{\boldsymbol{\gamma}}}_{e}} \tilde{\boldsymbol{\Phi}}_{\boldsymbol{i}, \boldsymbol{f}}+2 \tilde{\boldsymbol{\Phi}}_{\boldsymbol{i}, \boldsymbol{c}}{ }^{T} \frac{\partial \boldsymbol{K}_{\boldsymbol{c f}}}{\partial \tilde{\tilde{\gamma}}_{e}} \tilde{\boldsymbol{\Phi}}_{\boldsymbol{i}, \boldsymbol{f}}+ \\
& +\tilde{\boldsymbol{\Phi}}_{\boldsymbol{i}, \boldsymbol{c}}{ }^{T} \frac{\partial \boldsymbol{K}_{\boldsymbol{c c}}}{\partial \tilde{\tilde{\gamma}}_{e}} \tilde{\boldsymbol{\Phi}}_{\boldsymbol{i}, \boldsymbol{c}}+\boldsymbol{\nu}_{\mathbf{1}}^{T} \frac{\partial \boldsymbol{K}_{\boldsymbol{f f}}}{\partial \tilde{\tilde{\gamma}}_{e}} \tilde{\boldsymbol{\Phi}}_{\boldsymbol{i}, \boldsymbol{f}}+ \\
& +\boldsymbol{\nu}_{\mathbf{1}}^{T} \frac{\partial \boldsymbol{K}_{\boldsymbol{f c}}}{\partial \tilde{\tilde{\gamma}}_{e}} \tilde{\boldsymbol{\Phi}}_{\boldsymbol{i}, \boldsymbol{c}}+\boldsymbol{\nu}_{\mathbf{2}}{ }^{T} \frac{\partial \boldsymbol{K}_{\boldsymbol{c f}}}{\partial \tilde{\tilde{\gamma}}_{e}} \tilde{\boldsymbol{\Phi}}_{\boldsymbol{i}, \boldsymbol{f}}+ \\
& +\boldsymbol{\nu}_{\mathbf{2}}^{T} \frac{\partial \boldsymbol{K}_{\boldsymbol{c c}}}{\partial \tilde{\tilde{\gamma}}_{e}} \tilde{\boldsymbol{\Phi}}_{\boldsymbol{i}, \boldsymbol{c}}+\left(2 \tilde{\boldsymbol{\Phi}}_{\boldsymbol{i}, \boldsymbol{f}}^{T} \boldsymbol{K}_{\boldsymbol{f f}}+\right. \\
& +2 \tilde{\boldsymbol{\Phi}}_{\boldsymbol{i}, \boldsymbol{c}}{ }^{T} \boldsymbol{K}_{\boldsymbol{c f}}+\boldsymbol{\nu}_{\mathbf{1}}{ }^{T} \boldsymbol{K}_{\boldsymbol{f} \boldsymbol{f}}+ \\
& \left.+\boldsymbol{\nu}_{\mathbf{2}}{ }^{T} \boldsymbol{K}_{\boldsymbol{c f}}\right) \frac{\partial \tilde{\boldsymbol{\Phi}}_{i, f}}{\partial \tilde{\gamma}_{e}}-\boldsymbol{\nu}_{\mathbf{2}}^{T} \frac{\partial \boldsymbol{f}_{\boldsymbol{i}, \boldsymbol{c}}}{\partial \tilde{\tilde{\gamma}}_{e}}
\end{aligned}
$$

Requiring the terms that multiply $\frac{\partial \tilde{\boldsymbol{\Phi}}_{i, f}}{\partial \tilde{\bar{\gamma}}_{e}}$ and $\frac{\partial \boldsymbol{f}_{i, c}}{\partial \tilde{\bar{\gamma}}_{e}}$ to vanish we get:

$$
\left[\begin{array}{cc}
\boldsymbol{K}_{\boldsymbol{f f}} & \boldsymbol{K}_{\boldsymbol{f c}} \\
\mathbf{0} & \boldsymbol{I}
\end{array}\right]\left[\begin{array}{l}
\nu_{1} \\
\boldsymbol{\nu}_{2}
\end{array}\right]=\left[\begin{array}{c}
-2\left(\boldsymbol{K}_{\boldsymbol{f f}} \tilde{\boldsymbol{\Phi}}_{\boldsymbol{i}, \boldsymbol{f}}+\boldsymbol{K}_{\boldsymbol{f c}} \tilde{\boldsymbol{\Phi}}_{\boldsymbol{i}, \boldsymbol{c}}\right) \\
\mathbf{0}
\end{array}\right]
$$

Being the right hand side null because of Eq. (41), we conclude that $\boldsymbol{\nu}_{\mathbf{1}}=\mathbf{0}$ and $\boldsymbol{\nu}_{\mathbf{2}}=\mathbf{0}$, that substituted in Eq. (44) gives the sensitivities of the statically estimated modal stiffness in Eq. (28).

The sensitivities of the statically estimated modal mass can be obtained by first expressing $\tilde{m}_{m, i}$ following the partition in Eq. (41) and then adding the vanishing terms related to the static load case as in Eq. 437. Thus:

$$
\begin{aligned}
\widehat{\tilde{m}}_{m, i}= & \tilde{\boldsymbol{\Phi}}_{\boldsymbol{i}, \boldsymbol{f}}{ }^{T} \boldsymbol{M}_{\boldsymbol{f f}} \tilde{\boldsymbol{\Phi}}_{\boldsymbol{i}, \boldsymbol{f}}+2 \tilde{\boldsymbol{\Phi}}_{\boldsymbol{i}, \boldsymbol{c}}{ }^{T} \boldsymbol{M}_{\boldsymbol{c f}} \tilde{\boldsymbol{\Phi}}_{i, \boldsymbol{f}}+ \\
& +\tilde{\boldsymbol{\Phi}}_{\boldsymbol{i}, \boldsymbol{c}}{ }^{T} \boldsymbol{M}_{\boldsymbol{c c}} \tilde{\boldsymbol{\Phi}}_{\boldsymbol{i}, \boldsymbol{c}}+\boldsymbol{\mu}_{\mathbf{1}}{ }^{T}\left(\boldsymbol{K}_{\boldsymbol{f} f} \tilde{\boldsymbol{\Phi}}_{\boldsymbol{i}, \boldsymbol{f}}+\right. \\
& \left.+\boldsymbol{K}_{\boldsymbol{f c}} \tilde{\boldsymbol{\Phi}}_{\boldsymbol{i}, \boldsymbol{c}}\right)+\boldsymbol{\mu}_{\mathbf{2}}{ }^{T}\left(\boldsymbol{K}_{\boldsymbol{c f}} \tilde{\boldsymbol{\Phi}}_{\boldsymbol{i}, \boldsymbol{f}}+\right. \\
& \left.+\boldsymbol{K}_{\boldsymbol{c c}} \tilde{\boldsymbol{\Phi}}_{\boldsymbol{i}, \boldsymbol{c}}-\boldsymbol{f}_{\boldsymbol{i}, \boldsymbol{c}}\right)
\end{aligned}
$$

We now repeat the same procedure as before: we compute the sensitivities of Eq. (46) and we require the terms that multiply $\frac{\partial \tilde{\boldsymbol{\Phi}}_{i, f}}{\partial \bar{\gamma}_{e}}$ and $\frac{\partial \boldsymbol{f}_{i, c}}{\partial \bar{\gamma}_{e}}$ to vanish. We therefore obtain Eq. $(29)$, that allows to compute the adjoint vector $\boldsymbol{\mu}=\left[\boldsymbol{\mu}_{1}{ }^{T}, \boldsymbol{\mu}_{2}{ }^{T}\right]^{T}$, and we get also the final expression of the sensitivities of the statically estimated modal mass (Eq. (28)) by removing the eliminated terms.

\section{References}

Aage et al., 2017. Aage, N., Andreassen, E., Lazarov, B. S., and Sigmund, O. (2017). Giga-voxel computational morphogenesis for structural design. Nature, 550(7674):84-86.

Acar and Shkel, 2001. Acar, C. and Shkel, A. (2001). A design approach for robustness improvement of rate gyroscopes. In MSM, International Conference on Modeling and Simulation of Microsystems, 4, pages 80-83.

Acar and Shkel, 2009. Acar, C. and Shkel, A. (2009). MEMS Vibratory Gyroscopes. MEMS Reference Shelf. Springer US, Boston, MA.

Acar and Shkel, 2005. Acar, C. and Shkel, A. M. (2005). Structurally decoupled micromachined gyroscopes with post-release capacitance enhancement. Journal of $\mathrm{Mi}$ cromechanics and Microengineering, 15(5):1092-1101.

Bendsøe and Sigmund, 1999. Bendsøe, M. P. and Sigmund, O. (1999). Material interpolation schemes in topology optimization. Archive of Applied Mechanics (Ingenieur Archiv), 69(9-10):635-654.

Bendsoe and Sigmund, 2004. Bendsoe, M. P. and Sigmund, O. (2004). Topology Optimization, volume 95. Springer Berlin Heidelberg, Berlin, Heidelberg.

Benkhelifa et al., 2010a. Benkhelifa, E., Farnsworth, M., Tiwari, A., Bandi, G., and Zhu, M. (2010a). Design and optimisation of microelectromechanical systems: a review of the state-of-the-art. International Journal of Design Engineering, 3(1):41.

Benkhelifa et al., 2010b. Benkhelifa, E., Farnsworth, M., Tiwari, A., and Zhu, M. (2010b). Evolutionary Algorithms for Planar MEMS Design Optimisation: A Comparative Study. volume 284, pages 199-210.

Bernstein et al., 1993. Bernstein, J., Cho, S., King, A. Kourepenis, A., Maciel, P., and Weinberg, M. (1993). A micromachined comb-drive tuning fork rate gyroscope. [1993] Proceedings IEEE Micro Electro Mechanical Systems, pages 143-148.

Bourdin, 2001. Bourdin, B. (2001). Filters in topology optimization. International Journal for Numerical Methods in Engineering, 50(9):2143-2158.

Bruggi et al., 2016. Bruggi, M., Zega, V., and Corigliano, A. (2016). Optimization of auxetic structures for MEMS applications. 2016 17th International Conference on Thermal, Mechanical and Multi-Physics Simulation and Experiments in Microelectronics and Microsystems, EuroSimE 2016, pages $1-5$.

Bruns and Tortorelli, 2001. Bruns, T. E. and Tortorelli, D. A. (2001). Topology optimization of non-linear elastic structures and compliant mechanisms. Computer Methods in Applied Mechanics and Engineering, 190(26-27):34433459 .

Chen et al., 2012. Chen, F., Chang, H., Yuan, W., Wilcock, R., and Kraft, M. (2012). Parameter optimization for a high-order band-pass continuous-time sigma-delta modulator MEMS gyroscope using a genetic algorithm approach. Journal of Micromechanics and Microengineering, 22(10):105006

Clausen and Andreassen, 2017. Clausen, A. and Andreassen, E. (2017). On filter boundary conditions in topology optimization. Structural and Multidisciplinary Optimization, 56(5):1147-1155

Cook, 1995. Cook, R. D. (1995). Finite Element Modeling for Stress Analysis.

Díaaz and Kikuchi, 1992. Díaaz, A. R. and Kikuchi, N. (1992). Solutions to shape and topology eigenvalue optimization problems using a homogenization method. Int. J. Numer. Methods Eng., 35(7):1487-1502. 
Farnsworth et al., 2017. Farnsworth, M., Tiwari, A., and Zhu, M. (2017). Multi-level and multi-objective design optimisation of a MEMS bandpass filter. Applied Soft Computing Journal, 52(March):642-656.

Guest et al., 2004. Guest, J. K., Prévost, J. H., and Belytschko, T. (2004). Achieving minimum length scale in topology optimization using nodal design variables and projection functions. International Journal for Numerical Methods in Engineering, 61(2):238-254.

Haronian, 1995. Haronian, D. (1995). Maximizing microelectromechanical sensor and actuator sensitivity by optimizing geometry.

Jog, 2002. Jog, C. (2002). Topology design of structures subjected to periodic loading. J. Sound Vib., 253(3):687-709.

Kempe, 2011. Kempe, V. (2011). Inertial MEMS. Cambridge University Press, Cambridge.

Krog et al., 2004. Krog, L., Tucker, A., Kemp, M., and Boyd, R. (2004). Topology Optimisation of Aircraft Wing Box Ribs. In 10th AIAA/ISSMO Multidisciplinary Analysis and Optimization Conference, Reston, Virigina. American Institute of Aeronautics and Astronautics.

Kulygin et al., 2012. Kulygin, A., Kirsch, C., Schwarz, P., Schmid, U., and Seidel, H. (2012). Decoupled surface micromachined gyroscope with single-point suspension. Journal of Microelectromechanical Systems, 21(1):206-216.

Ma et al., 1994. Ma, Z.-D., Cheng, H.-C., and Kikuchi, N. (1994). Structural design for obtaining desired eigenfrequencies by using the topology and shape optimization method. Comput. Syst. Eng., 5(1):77-89.

Ma et al., 1995. Ma, Z.-D., Kikuchi, N., and Cheng, H.-C. (1995). Topological design for vibrating structures. Comput. Methods Appl. Mech. Eng., 121(1-4):259-280.

Olhoff, 1989. Olhoff, N. (1989). Multicriterion structural optimization via bound formulation and mathematical programming. Struct. Optim., 1(1):11-17.

Pedersen, 2000. Pedersen, N. (2000). Maximization of eigenvalues using topology optimization. Structural and Multidisciplinary Optimization, 20(1):2-11.

Philippine et al., 2013. Philippine, M. A., Sigmund, O., Rebeiz, G. M., and Kenny, T. W. (2013). Topology optimization of stressed capacitive RF MEMS switches. Journal of Microelectromechanical Systems, 22(1):206-215.

Poulsen, 2003. Poulsen, T. A. (2003). A new scheme for imposing a minimum length scale in topology optimization. International Journal for Numerical Methods in Engineering, 57(6):741-760.

Sahin et al., 2009. Sahin, K., Sahin, E., Alper, S. E., and Akin, T. (2009). A wide-bandwidth and high-sensitivity robust microgyroscope. Journal of Micromechanics and Microengineering, 19(7).

Sardan et al., 2008. Sardan, O., Petersen, D. H., Mølhave, K., Sigmund, O., and Bøggild, P. (2008). Topology optimized electrothermal polysilicon microgrippers. Microelectronic Engineering, 85(5-6):1096-1099.

Seyranian et al., 1994. Seyranian, A. P., Lund, E., and Olhoff, N. (1994). Multiple eigenvalues in structural optimization problems. Struct. Optim., 8(4):207-227.

Sigmund, 2001. Sigmund, O. (2001). Design of multiphysics actuators using topology optimization Part I:One-material structures. Computer Methods in Applied Mechanics and Engineering, 190(49-50):6605-6627.

Solouk et al., 2019. Solouk, M. R., Shojaeefard, M. H., and Dahmardeh, M. (2019). Parametric topology optimization of a mems gyroscope for automotive applications. Mechanical Systems and Signal Processing, 128:389 - 404.

Stolpe and Svanberg, 2001. Stolpe, M. and Svanberg, K. (2001). An alternative interpolation scheme for minimum compliance topology optimization. Structural and Multidisciplinary Optimization, 22(2):116-124.

Sung et al., 2011. Sung, W. K., Dalal, M., and Ayazi, F. (2011). A mode-matched 0.9 MHZ single proof-mass dualaxis gyroscope. 2011 16th International Solid-State Sensors, Actuators and Microsystems Conference, TRANSDUCERS'11, pages 2821-2824.

Svanberg, 1987. Svanberg, K. (1987). The method of moving asymptotes - A new method for structural optimization.

Svanberg, 2002. Svanberg, K. (2002). Methods Based on Conservative Convex Separable. Society, 12(2):555-573.

Tcherniak, 2002. Tcherniak, D. (2002). Topology optimization of resonating structures using SIMP method. Int. J. Numer. Methods Eng., 54(11):1605-1622.

Tenek and Hagiwara, 1993. Tenek, L. H. and Hagiwara, I. (1993). Static and vibrational shape and topology optimization using homogenization and mathematical programming. Comput. Methods Appl. Mech. Eng., 109(12):143-154.

Trusov et al., 2011. Trusov, A. A., Schofield, A. R., and Shkel, A. M. (2011). Micromachined rate gyroscope architecture with ultra-high quality factor and improved mode ordering. Sensors and Actuators, A: Physical, 165(1):2634 .

Vigna, 2011. Vigna, B. (2011). Tri-axial MEMS gyroscopes and six degree-of-freedom motion sensors. Technical Digest - International Electron Devices Meeting, IEDM, pages 662-664.

Wang et al., 2011. Wang, F., Lazarov, B. S., and Sigmund, O. (2011). On projection methods, convergence and robust formulations in topology optimization. Structural and Multidisciplinary Optimization, 43(6):767-784.

Xia et al., 2015. Xia, D., Kong, L., and Gao, H. (2015). Design and analysis of a novel fully decoupled tri-axis linear vibratory gyroscope with matched modes. Sensors (Switzerland), 15(7):16929-16955.

Xia et al., 2014. Xia, D., Yu, C., and Kong, L. (2014). The development of micromachined gyroscope structure and circuitry technology. Sensors (Switzerland), 14(1):13941473.

Zaman et al., 2006. Zaman, M., Sharma, A., and Ayazi, F. (2006). High Performance Matched-Mode Tuning Fork Gyroscope. In 19th IEEE International Conference on Micro Electro Mechanical Systems, number January, pages 66-69. IEEE.

Zhou et al., 2015. Zhou, M., Lazarov, B. S., Wang, F., and Sigmund, O. (2015). Minimum length scale in topology optimization by geometric constraints. Computer Methods in Applied Mechanics and Engineering, 293:266-282. 